\title{
An Inpainting- Based Deinterlacing Method
}

\author{
Coloma Ballester, Marcelo Bertalmío, Vicent Caselles, Associate Member, IEEE, Luis Garrido, \\ Adrián Marques, and Florent Ranchin
}

\begin{abstract}
Video is usually acquired in interlaced format, where each image frame is composed of two image fields, each field holding same parity lines. However, many display devices require progressive video as input; also, many video processing tasks perform better on progressive material than on interlaced video. In the literature, there exist a great number of algorithms for interlaced to progressive video conversion, with a great tradeoff between the speed and quality of the results. The best algorithms in terms of image quality require motion compensation; hence, they are computationally very intensive. In this paper, we propose a novel deinterlacing algorithm based on ideas from the image inpainting arena. We view the lines to interpolate as gaps that we need to inpaint. Numerically, this is implemented using a dynamic programming procedure, which ensures a complexity of $O(S)$, where $S$ is the number of pixels in the image. The results obtained with our algorithm compare favorably, in terms of image quality, with state-of-the-art methods, but at a lower computational cost, since we do not need to perform motion field estimation.
\end{abstract}

Index Terms-Geometry, interpolation, interlacing, inpainting, video restoration.

\section{INTRODUCTION}

$\mathbf{I}$ NTERLACED scanning is a sampling method for video sequences. In interlaced scanning, the scene is captured by sampling iteratively a set of odd and even fields. The even field holds the even lines of the frame, the odd field holds the odd lines of the frame. These fields are captured successively at regularly spaced time intervals. Since the invention of TV in the early 1930s, the interlaced scan has been adopted by many broadcasting systems such as PAL, NTSC, or SECAM, and can be considered the result of a tradeoff between vertical resolution and frame rate and transmission bandwidth requirements. Indeed, interlacing is a way of doubling the frame rate by halving the vertical resolution.

Early TV displays, based on cathode ray tube technology, were designed to work with an interlaced input. The low-pass filter response of the human eye and the one of the phosphor at

Manuscript received October 17, 2006; revised May 23, 2007. This work was supported in part by the IP-RACINE Project IST- 511316. C. Ballester, M. Bertalmío, and V. Caselles were supported by the PNPGC project, reference MTM2006-14836. M. Bertalmío and L. Garrido were supported by the Ramón y Cajal Program. V. Caselles was supported by the Departament d'Universitats, Recerca i Societat de la Informació de la Generalitat de Catalunya. The associate editor coordinating the review of this manuscript and approving it for publication was Dr. Dimitri Van De Ville.

The authors are with the Departament de Tecnologia, Universitat Pompeu Fabra, Barcelona, Spain (e-mail: coloma.ballester@upf.edu; marcelo.bertalmio@upf.edu; vicent.caselles@upf.edu; luis.garrido@upf.edu; adrian.marques@upf.edu; florent.ranchin@upf.edu).

Color versions of one or more of the figures in this paper are available online at http://ieeexplore.iee.org.

Digital Object Identifier 10.1109/TIP.2007.903844 the display allowed to reduce annoying effects due to the sampling method. Nowadays, many displays based on novel technologies such as liquid crystal or plasma do need a full frame as input. If these devices receive an interlaced signal, it must be converted to progressive before it can be displayed.

Interlaced-to-progressive conversion (IPC) approaches allow us to convert interlaced material into a progressive one. Progressive material is not only necessary for display in progressive devices, but also because some image processing tasks may require progressive video. IPC can be accomplished through a process that, for each field, doubles the vertical resolution using as input only the lines of the current field-intraframe reconstruction - or the lines of previous and following fields-interframe reconstruction.

There is a vast number of techniques in the field of IPC, techniques which can be based on intraframe reconstruction methods, interframe reconstruction methods, or a combination of both. There is an important tradeoff between speed and quality with these algorithms: the algorithms that introduce less visual artifacts require motion compensation (MC), which is a computationally very intensive procedure (implying the computation of motion vectors for every pixel). On the other hand, among the algorithms that do not require $\mathrm{MC}$, some of the best ones are directional interpolators, which make use of the edge information to decide, for each missing pixel, the spatial and/or temporal direction in which interpolation takes place. Directional interpolators have varied problems such as being sensitive to noise, only being able to detect a limited number of orientations or having problems to reconstruct periodic structures. More importantly, they all decide the interpolation direction on a very local basis, that is, the decision on the interpolation direction for each missing pixel is done independently of the decision done for neighboring pixels. This sometimes results in very noticeable visual artifacts.

Our main contribution in this paper is a novel deinterlacing algorithm that does not require MC though it may be complemented with it. Our approach is based on variational inpainting techniques [26], [27] and may be interpreted as a way of performing directional interpolation but with a global (instead of local) optimization criterion which takes into account the ordered structure of the level sets of the image. Our experiments show that it can be favorably compared both quantitatively and qualitatively with most MC deinterlacing techniques, which are the state-of-the-art algorithms in the literature.

The outline of the paper is as follows. In Section II, we review the most common strategies used for deinterlacing. In Section III, we review the basic idea involving the combination of several interpolation methods to produce a new and more robust one. In Section IV-A, we explain the main ideas behind geometric inpainting methods and we propose our deinterlacing 
method: we compute a spatial interpolation, a temporal interpolation and we combine both of them. In Section V, we display the results of our experiments and we compare our method with other algorithms proposed in the literature. We also compare our method with the combination of it with MC deinterlacing, showing that the gain in quality is very little. Our conclusions are summarized in Section VI.

\section{CURRENT IPC APPROACHES}

This section is devoted to a brief description of some of the methods available for IPC. They range from the very simple line averaging to more complex IPC based on MC. All the methods described here have been implemented in our paper in order to benchmark them against our proposal. In this paper, we divide the IPC methods into four classes: linear methods, directional interpolators, motion-adaptive IPC, and motion-compensated IPC. We will describe with some more detail the directional interpolators, since they are related to our approach.

To fix the notation, throughout this paper $I(\mathbf{p}, n)$ denotes an interlaced sequence, where $\mathbf{p}=(x, y) \in$ $\{1, \ldots, M\} \times\{1, \ldots, N\}$ corresponds to the pixel coordinates, and $n \in\{1, \ldots, T\}$ to the time sample $(N, M, T$ are positive integers). The size of $I$ corresponds to the size associated to the full progressive frame. Thus, we are assuming that in even fields of $I$ the even lines are defined whereas the odd lines are not, and that in odd fields of $I$ the odd lines are defined whereas the even lines are not. Notice that, as an interpolation process, the output of a deinterlacer is constructed by filling the values of the missing pixels using the known pixel values of $I$.

\section{A. Linear Methods}

These methods perform linear operations on the interlaced material in order to double the vertical resolution. The term linear stems from the fact that the interpolated pixel is obtained by a linear combination of spatiotemporal neighboring pixels. Some IPC methods that can be included in this class are as follows (see [35] and references therein).

- Line Doubling: As its name indicates, interpolation is performed by doubling each line. The resulting sequence's vertical resolution is half of the original and usually suffers from severe flickering.

- Line Average: Each missing pixel is interpolated by averaging the upper and lower pixel in the spatial direction, i.e., using the formula

$$
I_{l a}(x, y, n)=\frac{1}{2}(I(x, y-1, n)+I(x, y+1, n)) .
$$

The resulting sequence still suffers from annoying artifacts, specially in regions with fine vertical details.

- Field Weaving: It corresponds to the temporal version of the line doubling method. Each missing pixel is copied from the previous frame at the sample pixel coordinate, $I_{f w}(\mathbf{p}, n)=I(\mathbf{p}, n-1)$. Field weaving allows perfect reconstruction of the missing lines for static areas, but very annoying artifacts may appear in moving areas.
- Field Averaging: It is the temporal analogous of line averaging. In this case the missing pixels are interpolated linearly between neighboring temporal samples

$$
I_{f a}(\mathbf{p}, n)=\frac{1}{2}(I(\mathbf{p}, n-1)+I(\mathbf{p}, n+1)) .
$$

Similar effects as for field weaving may appear with this technique.

- Vertical-Temporal (VT) Filtering: In this case, the impulse response of the filter has support in the space-time domain and is designed to avoid motion artifacts, but it is difficult to keep vertical detail when high temporal frequencies are present (see [13] and references therein).

\section{B. Directional Interpolation Methods}

These methods try to use the edge information present in the scene for a proper reconstruction of the progressive frame. For each missing pixel, the neighboring spatiotemporal pixels are analyzed and a decision on the direction in which interpolation should be done is taken. Some of the IPC methods that are included into the class of directional interpolators are as follows.

- Edge-Based Line Average (ELA) [14]: For each missing pixel, an estimation of the direction of the edge passing through it is performed based on the correlation of luminance values of the $3 \times 3$ window centered around it. If $(x, y)$ is the pixel to interpolate, the interpolation direction is estimated by evaluating the three pixel differences $|I(x, y-1)-I(x, y+1)|,|I(x-1, y-1)-I(x+1, y+1)|$, and $|I(x+1, y-1)-I(x-1, y+1)|$. The pixel $(x, y)$ is then linearly interpolated between the pair of pixels that results in the lowest pixel difference.

Compared to linear approaches, ELA may reconstruct sharply oblique lines, avoiding many of the jagged edges produced by line doubling or line averaging. However, ELA still produces noticeable flicker. Moreover, due to the very limited number of directions considered with this algorithm, ELA may incorrectly estimate the edge direction, thereby assigning to the missing pixel a value that stands out as incorrect and is, thus, also clearly visible.

- Direction Oriented Interpolation [40] (DOI): This approach tries to determine the orientation of the edge at a missing pixel in a more robust manner than ELA does. For that purpose, instead of computing simple pixel differences to estimate the edge orientation as ELA does, a set of windows centered at the corresponding pixels are used. As shown in Fig. 1, three windows are used: one window $W_{0}$ centered at the missing pixel, and two sliding windows $W_{1}$ and $W_{2}$. By comparing the pixels of $W_{0}$ and $W_{1}$, and the pixels of $W_{0}$ and $W_{2}$, the edge direction can be determined, and, thus, the missing pixel may be interpolated. Even if DOI is more robust than ELA, our experiments show that DOI may produce annoying artifacts in periodic structures.

- Spatiotemporal ELA [29] (STELA): This directional interpolator evaluates six different directional changes in the spatiotemporal direction as shown in Fig. 2. The interpolated value is obtained by taking the median over a set of 


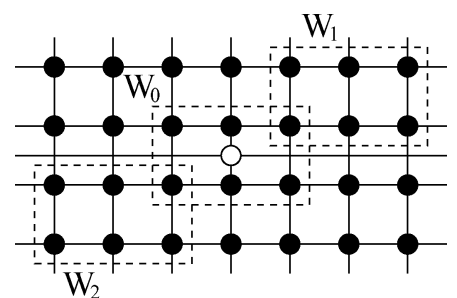

Fig. 1. Reference windows in DOI approach.

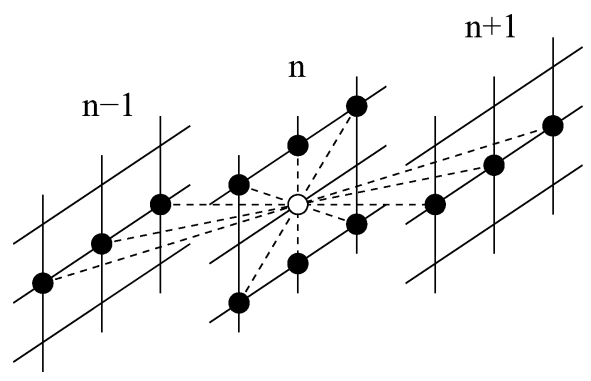

Fig. 2. STELA spatiotemporal window.

values obtained from the pixel differences. STELA outperforms the previously presented directional interpolators in slow or static areas since it is exploiting temporal information in addition to the spatial one to which the ELA and DOI are restricted.

- Structure Tensor Edge Orientation Estimation [36]: In [36], the authors considered the problem as an inpainting problem and proposed a spatial interpolation along the edge directions, which were estimated in a robust way using the structure tensor, combined with a MC interpolation using the result of the first spatial step.

Many other variants of these ideas have been proposed (see [15], [23], and [31]).

\section{Motion Adaptive Methods}

Spatial deinterlacing techniques exploit the correlation between neighboring samples in a field and, thus, work properly in the case that there are no vertical details appearing. Temporal deinterlacing techniques exploit the correlation in the temporal domain and, thus, work properly in the absence of motion.

Motion adaptive methods aim at exploiting the best of both types of methods. As we have said, their performance depends on the presence or absence of motion. For instance, field weaving behaves well in areas where no motion is present whereas line averaging behaves better than weaving in moving areas. Thus, the main issue in motion adaptive IPC methods is the robust detection of moving parts of the image.

Motion detection is usually based on field difference [8], [22], [34]. Two well known motion detectors for IPC are depicted in Fig. 3. The first, shown on the left, evaluates the presence of motion using two fields: for each pixel $X$, it computes the difference $|A-B|$. The second, shown on the right, uses four fields to detect motion: for each pixel $X$ three differences, $\mid A-$ $B|| C-D \mid$, , and $|E-F|$ are computed.

Based on this field difference, a hard or soft decision on the presence of motion may be taken with the idea of fading be-
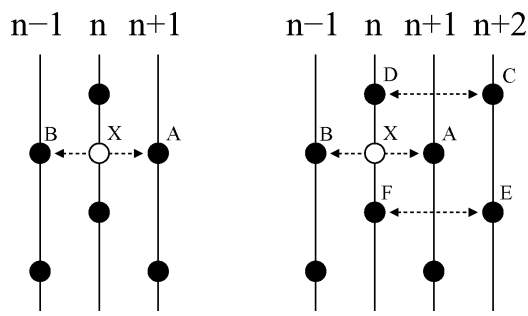

Fig. 3. Illustration of different motion detectors.

tween two interpolators, each one adapted to the presence or absence of motion [1], [5]. In other cases, several interpolators are combined depending on the frequency content, the presence or absence of motion [17]. On the other hand, nonlinear filters, like the median filter, when computed on a suitable spatiotemporal neighborhood, implicitly adapt to motion or edges [19], [29], [30]. Moreover, it can be combined with other methods to adapt to the type of motion, slow or fast [32].

In [20], the authors switch between temporal average and edge dependent interpolation according to motion and edge detectors. In the case of edge oriented interpolation, the edge direction is determined by minimizing a distance between neighborhoods (of the low-pass-filtered versions of the image). The method proposed below is also related to these ideas.

\section{Motion-Compensated Methods}

Motion estimation provides the IPC with a new spatiotemporal direction along which the image may be interpolated. Motion-compensated IPC methods make use of the motion vector field information to enhance interpolation results. The quality of the IPC interpolation depends on the accuracy of the motion vector fields. Due to the ill-posed nature of the motion estimation process, it is not affordable to obtain accurate motion vectors everywhere. Thus, the IPC has to be able to detect when motion vectors have been incorrectly estimated.

Some motion-compensated IPC methods are listed as follows.

- Motion-Compensated Field Weaving [37]: It is a time recursive algorithm: the output of the interlacer at field $n$, denoted by $I_{d}(\mathbf{p}, n)$, is computed by motion compensating the previous deinterlaced field $n-1$ onto the current one, $I_{d}(\mathbf{p}, n)=I(\mathbf{p}-\mathbf{d}(\mathbf{p}, n) n-1)$, where $\mathbf{d}$ is the estimated motion field for pixel $\mathrm{p}$. This IPC method lacks from a protection against incorrectly estimated motion vectors, and, thus, annoying artifacts may appear on those areas.

- Motion Compensated-Motion Adaptive [39]: If motion has been accurately estimated, MC virtually converts a moving sequence into a nonmoving one. Moving areas in the motion-compensated sequence may be due to incorrect motion vectors. Thus, a motion adaptive scheme may prove useful here. After MC, the motion adaptive scheme detects the moving areas, that is, areas where motion has not been properly compensated. Moving areas may then be corrected with, for instance, a spatial interpolator.

- Adaptive Recursive Motion Compensation [12]: In [12], the authors proposed a motion-compensated first-order recursive temporal filter: as inputs it requires the motion 


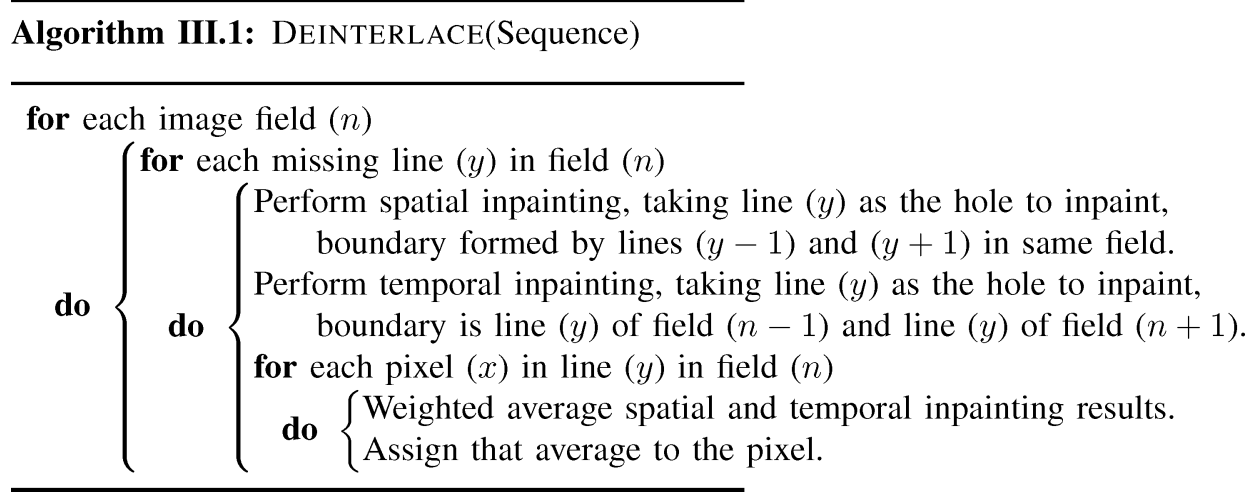

Fig. 4. Pseudocode for the deinterlacing algorithm proposed in this paper.

field $\mathbf{d}(\mathbf{p}, n)$ and the output $I_{i}(\mathbf{p}, n)$ of an initial deinterlacing algorithm (like line averaging). Assuming that the previous field $n-1$ has already been deinterlaced, i.e., we have $I_{d}(\mathbf{p}, n-1)$, then the deinterlaced value at $(\mathbf{p}, n)$ is computed as a weighted averaging of the values of $I_{i}(\mathbf{p}, n)$ (resp., $\left.I(\mathbf{p}, n)\right)$ and $I_{d}(\mathbf{p}-\mathbf{d}(\mathbf{p}, n), n-1)$ if $y \bmod 2=(n-1) \bmod 2(\operatorname{resp} ., y \bmod 2=n \bmod 2)$. The weighting parameters are adaptive and are designed so as to reduce nonstationarities along the motion trajectory.

\section{E. Discussion}

As can be seen, there exist a large pool of approaches for IPC. Our experiments show that the best results are obtained with motion-compensated methods, which are computationally quite intensive.

On the other hand, and as we described above, the directional interpolators decide the interpolation direction using the values of neighboring pixels of the missing pixel. However, there is no constraint for edge directions of neighboring pixels and edges may cross. This is, according to our experiments, the main reason why these methods fail to properly reconstruct the missing pixels.

Our purpose in this paper is to develop a new and robust directional interpolator that takes into account the fact that level lines of the image do not cross. This geometric property is imposed as a global optimization constraint. With this, most of the annoying artifacts of local directional interpolators are avoided. Moreover, our interpolator is applied both in the spatial and temporal domain and, as a result, our IPC does not need to rely on $\mathrm{MC}$ to obtain good results.

\section{COMbining Several InTERPOLATION MethodS}

The combination of several IPC methods proposed in [12] leads to an improved result. In a further development of this idea, in [21], Kovačević et al. proposed an algorithm for deinterlacing video sequences by weighting several interpolation methods. The basic idea is to combine several interpolation methods in a recursive way using successive approximations.

The first level of approximation [21] consists in combining the result of two simple interpolation methods, line and field averaging methods $I_{l a}, I_{f a}$ using a linear combination with weights $k_{l a}(\mathbf{p}, n), k_{f a}(\mathbf{p}, n) \geq 0$ which depend on the reliability of the method

$$
I_{l f a}(\mathbf{p}, n)=k_{l a}(\mathbf{p}, n) I_{l a}(\mathbf{p}, n)+k_{f a}(\mathbf{p}, n) I_{f a}(\mathbf{p}, n) .
$$

where $k_{l a}(\mathbf{p}, n)+k_{f a}(\mathbf{p}, n)=1$. The weight $k_{l a}(\mathbf{p}, n)$ [resp., $\left.k_{f a}(\mathbf{p}, n)\right]$ is larger when the neighborhoods of pixels $(x, y-$ $1, n)$ and $(x, y+1, n)$ [resp., of pixels $(\mathbf{p}, n-1)$ and $(\mathbf{p}, n+1)]$ are similar where the similarity of two neighborhoods is measured by a (weighted) $\ell^{2}$ distance. In other words, the coefficients are an increasing function of the reliability of the method, the reliability being an increasing function of the similarity [see the weights in formula (5)]. We shall keep this basic rule in what follows and in our deinterlacing method below.

In the second level of approximation [21], the result of the first level $I_{l f a}(\mathbf{p}, n)$ is used to compute the edge direction (using steerable filters) on pixel $\mathbf{p}$ of frame $n$ and then compute an edge-based line average interpolation $I_{e a}$. Then the authors combine the results $I_{l a}, I_{f a}$ and $I_{e a}$ using a weighted average where the weight given to each term depends on its reliability. Let $I_{l f e}$ be the image obtained.

The third level of approximation [21] uses $I_{l f e}$ to compute a forward and backward motion estimation $\mathbf{v}^{f}=\left(v_{x}^{f}, v_{y}^{f}\right), \mathbf{v}^{b}=$ $\left(v_{x}^{b}, v_{y}^{b}\right)$, which are used to interpolate a new image in a recursive way

$$
\begin{aligned}
I_{m c}(\mathbf{p}, n)=k_{b}(\mathbf{p}, n) I_{m c}\left(\mathbf{p}-\mathbf{v}^{b}\right. & , n-1) \\
& +k_{f}(\mathbf{p}, n) I_{l f e}\left(\mathbf{p}-\mathbf{v}^{f}, n+1\right)
\end{aligned}
$$

where $k_{f}(\mathbf{p}, n), k_{b}(\mathbf{p}, n)$ are weight factors which depend on the reliability of the motion estimates. The final deinterlaced sequence is obtained as a weighted average of the images $I_{l a}$, $I_{f a}, I_{e a}, I_{m c}$ with coefficients depending on the reliability of the estimate, giving more weight to the more reliable ones (see [21]).

Our purpose is to follow the same successive approximation approach using only two steps: in the first step we compute an inpainting-based (intraframe) deinterlacing, in the second step we compute a motion adaptive interpolation (interframe), and the weighted average of these two interpolations gives more weight (locally at each pixel) to the more reliable one. The overall algorithm is illustrated in the pseudocode of Fig. 4. In practice, we have observed that if we further refine the restoration by means of a MC procedure, the quality increase is almost unnoticeable. 


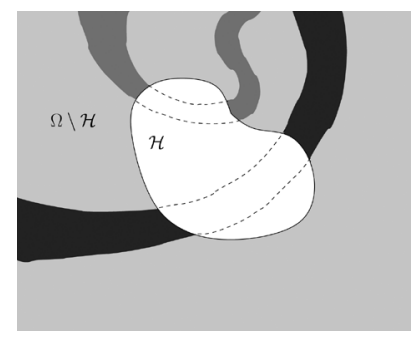

Fig. 5. Image with a hole $\mathcal{H}$ to be inpainted. Following Masnou and Morel [26], [27], the level lines in $\Omega \backslash \mathcal{H}$ are continued inside the hole by interpolating them with an elastica type energy.

\section{INPAINTING-BASED METHOD}

\section{A. Review of Some Basic Inpainting Ideas}

Our purpose in this section is to review some inpainting methods and stress some ideas that we shall use in our deinterlacing method. To explain these ideas in a general context, not necessarily related to deinterlacing, we consider a 2-D digital image, and we denote it by $u:\{1, \ldots, M\} \times\{1, \ldots, N\} \rightarrow\{1, \ldots, L\}$. To shorten our notation, we write $\Omega:=\{1, \ldots, M\} \times\{1, \ldots, N\}$.

In their paper [26], [27], Masnou and Morel proposed an inpainting method based on the reconstruction of the family of level lines of the image in the region where the information is missing. Recall that the upper (resp., lower) level sets of the image $u: \Omega \rightarrow\{1, \ldots, L\}$ are the sets

$$
\begin{gathered}
\{(x, y) \in \Omega: u(x, y) \geq \lambda\} \\
(\operatorname{resp} .\{(x, y) \in \Omega: u(x, y)<\lambda\})
\end{gathered}
$$

where $\lambda \in\{1, \ldots, L\}$. We call level lines the boundaries of the connected components of level sets. Using the right notion of connectivity (8-connectedness for upper level sets and 4-connectedness for lower level sets), the level lines do not cross each other.

Suppose that $\mathcal{H}$ is a region of the image domain where the information is missing, which we will call a hole. The proposal in [26] and [27] was to reconstruct the level lines of the image $u$ inside the hole $\mathcal{H}$ knowing them in the complementary region $\Omega \backslash \mathcal{H}$ (see Fig. 5). Once we have reconstructed the level lines inside $\mathcal{H}$ and knowing the image $u$ in $\Omega \backslash \mathcal{H}$, we may reconstruct the corresponding level sets in $\mathcal{H}$, denote them by $X^{\lambda}$, as the regions determined by them (the computational process must guarantee that this is possible) and then reconstruct the image by the classical formula of mathematical morphology

$$
u(x, y)=\max \left\{\lambda \in\{1, \ldots, L\}:(x, y) \in X^{\lambda}\right\}, \quad(x, y) \in \mathcal{H} \text {. }
$$

To be able to use this formula, the reconstructed level sets must satisfy the basic inclusion property of level sets, i.e., if $\lambda \geq \mu$, then $X^{\lambda} \subset X^{\mu}$. This amounts to say that level lines do not cross. Thus, to follow this program we need to impose this property as done by the authors of [25] and [27].

To interpolate the missing level lines by means of short and not too oscillating curves, Masnou and Morel's proposal was to use the elastica which is the curve $\Gamma$ that joins two points $p, q$ having specified tangents at them and minimizes $\int_{\Gamma}(\alpha+$ $\left.\beta|\kappa|^{p}\right) d s$, with $p=2, \alpha, \beta>0$, where $\kappa$ denotes the curvature of $\Gamma$ and $d s$ its arc-length element. If $\mathcal{L}^{\lambda}$ denotes the level line corresponding to the boundary of the level set $\lambda$ of $u$ in $\Omega \backslash$ $\mathcal{H}$, i.e., the boundary of $\{(x, y) \in \Omega \backslash \mathcal{H}: u(x, y) \geq \lambda\}$, the authors of [25] and [27] proposed to compute the elastica's completion of $\mathcal{L}^{\lambda}$ inside $\mathcal{H}$. The precise conditions ensuring that this is possible were analyzed in [25].

The optimal disocclusion requires to compute the continuation of all level lines $\mathcal{L}^{\lambda}$ for any $\lambda$. To accomplish this, the authors add the elastica energy of all these completions [25] and [27] to obtain the final energy functional that is minimized.

Finally, in [25] and [26], choosing the power $p=1$ and relaxing the tangent conditions at end points, the authors proposed an algorithm based on dynamic programming to find optimal pairing between compatible points in $\partial \mathcal{H}$ obtaining a minimum of the energy (whose existence was also proved). Compatible points are points in $\partial \mathcal{H}$ with the same gray level and with the same gradient orientation and they are candidates to be joined by curves completing the level lines inside $\mathcal{H}$. Moreover, an admissible solution should satisfy the property that two level lines connecting two pairs of compatible points should not cross. Then, in order to compute the optimal solution, it is enough to enumerate all possible connections between points in $\partial \mathcal{H}$ satisfying the above requirements, compute the connecting curves (which are straight lines when $p=1$ ) and their energy and keep the one with smallest energy. However, thanks to the fact that every admissible solution satisfies a causality principle in the sense that every association between two points coerces any new association (so that no crossing of level lines is produced), it is possible to design a dynamic programming algorithm whose time complexity is at most cubic in the number of pixels of $\partial \mathcal{H}$ [25], [26].

The previous method has a good performance when applied to reconstruct the geometric sketch of an image, but is not adapted to texture recovery. In [16], Efros and Leung introduced a nonparametric technique for texture synthesis that has been later adapted for image inpainting [4], [11]. Texture is modeled as a Markov random field and the authors assume that the brightness value of a pixel given its spatial neighborhood is independent of the rest of the image. With this assumption, the conditional distribution of the brightness value of a pixel $\mathbf{p}$ given its spatial neighborhood $\mathcal{N}(\mathbf{p})$ (which is taken as a squared window around $\mathbf{p}$ ) is estimated by querying the sample image and finding its similar neighborhoods. The texture synthesis algorithm grows a new image, one pixel at a time. Given a sample image, to synthesize the value of a new pixel $\mathbf{q}$, the algorithm finds the neighborhoods of the sample image which are similar to the neighborhood of $\mathbf{q}$ and randomly chooses one of them and takes the value of its center as the new synthesized value at q. When applied to image inpainting the sample image is given by the known part of the image, i.e., $\Omega \backslash \mathcal{H}$, and the algorithm is applied to synthesize the values of the image inside the hole $\mathcal{H}$. To synthesize the value of pixel $\mathbf{q}$ in $\partial \mathcal{H}$ (i.e., points in $\mathcal{H}$ with a neighbor in $\Omega \backslash \mathcal{H}$ ), we compare the known part of its neighborhood $\mathcal{N}(\mathbf{q}) \backslash \mathcal{H}$ with the neighborhood $\mathcal{N}(\mathbf{p})$ of points $p \in \Omega \backslash \mathcal{H}$ by using a weighted $\ell^{2}$ distance

$$
\begin{aligned}
d_{2}^{w}(\mathcal{N}(\mathbf{q}), \mathcal{N}(\mathbf{p})) & \\
= & \sum_{\mathbf{h}: \mathbf{q}+\mathbf{h} \in \mathcal{N}(\mathbf{q}) \backslash \mathcal{H}} w(\mathbf{h})|u(\mathbf{q}+\mathbf{h})-u(\mathbf{p}+\mathbf{h})|^{2}
\end{aligned}
$$

where $w(\mathbf{h})$ is a weighting kernel (like a Gaussian kernel). Similar neighborhoods are those which almost realize the min- 
imum distance to $\mathcal{N}(\mathbf{q}) \backslash \mathcal{H}$ and the distribution of its center values give a histogram for the values of $\mathbf{p}$ which can then randomly sampled [16]. When a new pixel value is synthesized the hole is redefined by excluding this pixel. Initially, the order of the pixels being synthesized was the standard concentric layer filling, but this was improved in [11] by giving more priority to pixels which are on the continuation of strong edges and are surrounded by high-confidence pixels (they have larger known parts in their neighborhoods), combining in such a way geometry and texture inpainting. In both cases [11], [16], a greedy approach for hole filling is used. In [38], the authors proposed a coherence measure to be optimized which tries to find for each pixel to be reconstructed the most similar one (in terms of its neighborhood) in the sample image and they applied it to image and video completion. What is of interest to us here is that they proposed to compute the unknown pixel value $u(\mathbf{q})$ by a weighted average of the values of pixels close enough to $\mathbf{q}$ in terms of $d_{2}^{w}$

$$
u(\mathbf{q})=\frac{1}{Z} \sum_{\mathbf{p}} u(\mathbf{p}) e^{-\frac{d_{2}^{w}(\mathcal{N}(\mathbf{q}), \mathcal{N}(\mathbf{p}))}{2 \sigma^{2}}}
$$

where the sum is extended to pixels with similar neighborhoods to $\mathcal{N}(\mathbf{q})$, the weight $e^{-\left(d_{2}^{w}(\mathcal{N}(\mathbf{q}), \mathcal{N}(\mathbf{p})) / 2 \sigma^{2}\right)}$ is a decreasing function of its argument expressing the reliability of the matching, the value of $\sigma$ is chosen empirically depending on the image noise, and $Z$ is a normalization factor, i.e, $Z=\sum_{\mathbf{p}} e^{-\left(d_{2}^{w}(\mathcal{N}(\mathbf{q}), \mathcal{N}(\mathbf{p})) / 2 \sigma^{2}\right)}$ [38]. The paper in [28], which was pointed out to us by one of the reviewers, is devoted to full-frame video stabilization using video completion and deblurring algorithms and contains several interesting ideas that could have some application in the context of deinterlacing. In particular, if motion estimation (using neighboring frames) produces a consistent estimate of a pixel value in a hole of a video sequence, then we can adopt it; otherwise, the authors propose to use a technique based on motion inpainting combined with other spatial intraframe inpainting methods.

\section{B. Inpainting-Based Method for Space Interpolation}

We propose to combine the two main ideas described in Section IV-A for intraframe interpolation in an interlaced video sequence $I(\mathbf{p}, n)$. Given a fixed frame $I(\mathbf{p})$, let us consider two consecutive lines of the same parity $(x, y-1)$ and $(x, y+1)$, $x \in\{1, \ldots, M\}$, where the image $I$ is known and let us consider the unknown line $(x, y), x \in\{1, \ldots, M\}$, as the hole to be inpainted. We denote these lines by $\mathcal{L}_{-}, \mathcal{L}_{+}$, and $\mathcal{L}_{0}$, respectively. According to Masnou and Morel's approach, since the level lines of the image do not cross, the connection of level lines between $\mathcal{L}_{-}$and $\mathcal{L}_{+}$, or, what amounts to the same, the reconstruction of the image $I$ in the missing line $\mathcal{L}_{0}$ defines a nondecreasing correspondence between points in $\mathcal{L}_{-}$and $\mathcal{L}_{+}$. On the other hand, due to the inclination of level lines and the possible presence of an object in one line and not in the other, the correspondence $\varphi$ is in general multivalued (see Fig. 6). Combining these two observations, we define $\varphi$ as a nondecreasing multivalued map, that is, a map which assigns to each $j \in \mathcal{L}_{-}$ an interval $\varphi(j)=\left[a_{j}, b_{j}\right] \subseteq \mathcal{L}_{+}$and such that:
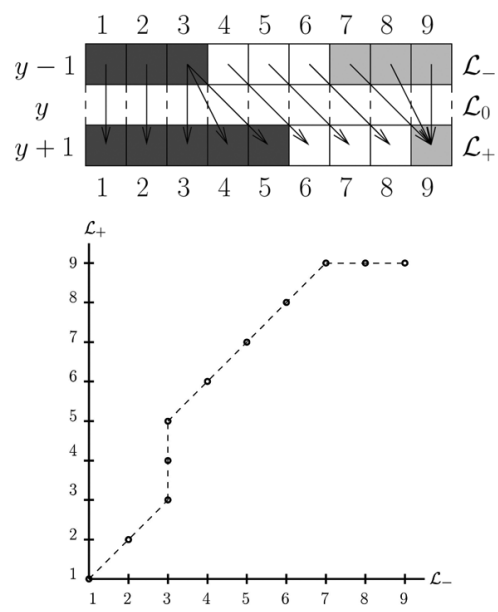

Fig. 6. (Top) Example of a nondecreasing warping expressing the correspondences between two lines $\mathcal{L}_{-}$and $\mathcal{L}_{+}$. Observe that a point may be mapped to an interval. (Bottom) Graph of the warping in top image.

a) the image of $\varphi$ covers all $\mathcal{L}_{+}$, i.e.,

$$
\cup_{j \in \mathcal{L}_{-}} \varphi(j)=\mathcal{L}_{+}
$$

and

b) $\varphi$ is nondecreasing in the sense that if $j<j^{\prime}$, the interval $\varphi(j)=\left[a_{j}, b_{j}\right]$ is located at the left of $\varphi\left(j^{\prime}\right)=\left[a_{j^{\prime}}, b_{j^{\prime}}\right]$, i.e, $b_{j} \leq a_{j^{\prime}}$.

We call such a mapping a nondecreasing warping and we denote by $\mathcal{W}\left(\mathcal{L}_{-}, \mathcal{L}_{+}\right)$the set of nondecreasing warpings from $\mathcal{L}_{-}$onto $\mathcal{L}_{+}$. Notice that the inverse of a nondecreasing warping from $\mathcal{L}_{-}$onto $\mathcal{L}_{+}$is also a nondecreasing warping, now from $\mathcal{L}_{+}$onto $\mathcal{L}_{-}$.

As we can see in Fig. 6, the graph of a nondecreasing warping has vertical walls (which would be discontinuities if the mapping was single valued) at the points $j \in \mathcal{L}_{-}$where $\varphi(j)$ is an interval. Both the vertical walls and the flat parts of the graph are related to the inclination of level lines and possible presence of an object in one line and not in the other (in the applications of this technique to compute correspondences in pairs of stereo images this is related to the presence of occlusions and disocclusions).

Given $\mathcal{L}_{+}$and $\mathcal{L}_{-}$, the nondecreasing warping $\varphi \in$ $\mathcal{W}\left(\mathcal{L}_{-}, \mathcal{L}_{+}\right)$must be chosen to connect similar structures in both lines. For that, we shall use the ideas coming from the texture synthesis technique of Efros and Leung, in that we shall connect points which have similar neighborhoods. Let us translate this into an energy functional. The cost associated to a nondecreasing warping $\varphi \in \mathcal{W}\left(\mathcal{L}_{-}, \mathcal{L}_{+}\right)$is given by the sum of the costs of the matchings

$$
\mathcal{C}(\varphi)=\sum_{x^{-} \in \mathcal{L}_{-}, x^{+} \in \varphi\left(x^{-}\right)} C\left(x^{-}, x^{+}\right)
$$

where $C\left(x^{-}, x^{+}\right)$is the cost of matching pixels $\left(x^{-}, y-1\right)$ and $\left(x^{+}, y+1\right)$. 

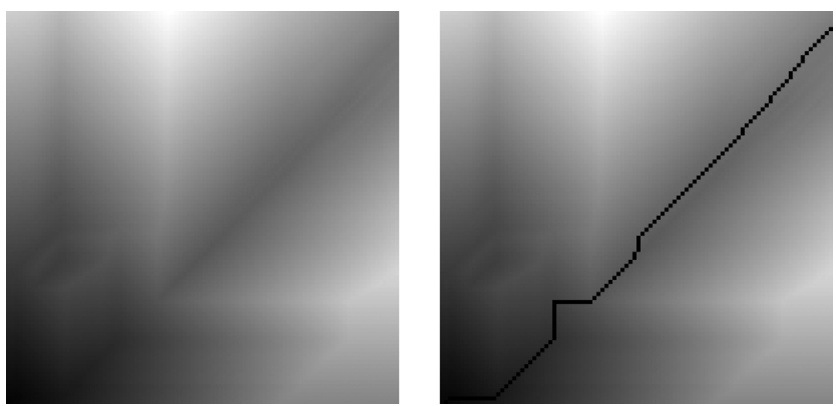

Fig. 7. Left: Cost matrix $C$ for lines 54 and 56 of the Highway image shown in Fig. 11. Light gray value $C(i, j)$ corresponds to a high cost of machtcing pixel $i$ in line 54 with pixel $j$ in line 56. Right: Best warping is shown superimposed in black; this optimum path is computed very fast via dynamic programming.

The optimal nondecreasing warping between $\mathcal{L}_{-}$and $\mathcal{L}_{+}$is obtained as the solution of

$$
\operatorname{Minimize}\left\{\mathcal{C}(\varphi): \varphi \in \mathcal{W}\left(\mathcal{L}_{-}, \mathcal{L}_{+}\right)\right\}
$$

A similar approach has been used in [10] for computing correspondences in epipolar lines in stereo images. The authors propose a Bayesian approach which leads to a similar functional. ${ }^{1}$ The optimum in (8) is computed using a dynamic programming algorithm. After computing the matrix of costs whose $(i, j)$ entry is the cost associated to each correspondence between the pixels $i \in \mathcal{L}_{-}$and $j \in \mathcal{L}_{+}$, the algorithm proposed in [10] computes the nondecreasing warping that minimizes (7). The warping is represented as a nondecreasing path in matrix $\mathcal{C}$ and is a list of pixel pairs whose gray values match. As we shall comment in our next paragraph, for computational reasons, we may restrict the choice of the optimal path to lie on a band around the diagonal (which represents the trivial warping $j \rightarrow j$ ).

Fig. 7 shows, on the left, the central diagonal band of the $100 \times 100$ cost matrix $\mathcal{C}$ for matching lines 54 and 56 of the Highway image shown in Fig. 11. In Fig. 11, a light gray value corresponds to a high cost, and a dark gray value corresponds to a low cost. Therefore, finding the optimum warping between these lines amounts to finding the path in this matrix (from the bottom left to the top right) which has a minimum accumulated cost. In general, finding the solution to this optimization problem has a complexity of $O\left(M^{3}\right)$, where $M$ is the number of pixels of each line. If we now introduce the ordering constraint, ${ }^{2}$ the complexity reduces to $O\left(M^{2}\right)$. As we see (Fig. 7, right), the optimal path does not deviate substantially from the diagonal, allowing us to compute just a diagonal band instead of the whole matrix. Restricting the optimal path to such a band reduces the complexity to $O(D M)$ where $D$ is the width of the band around the diagonal of the matrix $\mathcal{C}$. Since, in practice, a small value relative to the image size of $D$ suffices, we have that the complexity of finding a warping between two lines is $O(M)$.

\footnotetext{
${ }^{1}$ Indeed, the cost in [10] corresponds to the choice $\mu=\beta=0$ in (9), as it will be shown later.

${ }^{2}$ This constraint states that if pixel $a$ is before pixel $b$ in line $\mathcal{L}_{-}$, then their correspondent matches in line $\mathcal{L}_{+}$, pixels $a^{\prime}$ and $b^{\prime}$, will also satisfy that $a^{\prime}$ comes before $b^{\prime}$.
}

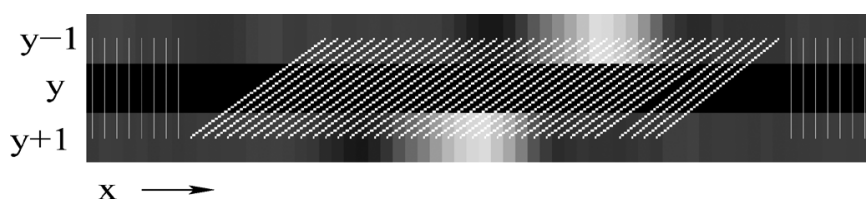

Fig. 8. Example of a nondecreasing warping $\varphi$ mapping from points in $\mathcal{L}_{-}$to ranges (points or intervals) in $\mathcal{L}_{+}$. The two lines $y-1$ and $y+1$ have been extracted from Fig. 11 and the matching has been computed by minimizing the $\operatorname{cost} \mathcal{C}$ described in Section IV-B. Observe that the computed warping is indeed multivalued.

Fig. 8 displays an example a nondecreasing warping between two lines of the image displayed in Fig. 11. The warping has been computed using the algorithm described in Section IV-B. The figure displays some pixels that are mapped in a one-to-one way while others are mapped to a single pixel and one is mapped to an interval of pixels.

To define the cost $C\left(x^{-}, x^{+}\right)$, we shall take into account the following items.

1) We favor the connection of points with similar neighborhoods.

2) We favor short connections against longer ones.

3) We shall avoid introducing new discontinuities in the interpolated image.

4) We shall penalize the number of "discontinuities" or vertical walls and the number of flat intervals in the graph of $\varphi$.

The first two items suggest to use the following cost for matching $x^{-}$to $x^{+}$:

$$
C^{1}\left(x^{-}, x^{+}\right)=\left(\alpha+\beta\left|x^{-}-x^{+}\right|\right) C_{\text {sim }}\left(x^{-}, x^{+}\right)
$$

where $\alpha, \beta>0$

$$
C_{\text {sim }}\left(x^{-}, x^{+}\right):=\sum_{\mathbf{h} \in \mathcal{N}^{*}(\mathbf{0})} w(\mathbf{h})\left|I\left(\mathbf{p}^{-}+\mathbf{h}\right)-I\left(\mathbf{p}^{+}+\mathbf{h}\right)\right|
$$

$w(\mathbf{h})$ is a weighting function, $\mathbf{p}^{-}=\left(x^{-}, y-1\right), \mathbf{p}^{+}=\left(x^{+}, y+\right.$ 1), $\mathcal{N}^{*}(\mathbf{0})$ being the intersection of $\mathcal{N}(\mathbf{0})$ with the known lines, and $\mathcal{N}(\mathbf{0})$ is a square neighborhood around $\mathbf{0}$, so that $\mathbf{p}+\mathbf{h}$ describes a square neighborhood around $\mathbf{p}$ when $\mathbf{h} \in \mathcal{N}(\mathbf{0})$. Thus, $C_{\text {sim }}\left(x^{-}, x^{+}\right)$is a weighted $\ell^{1}$ distance function between the neighborhoods of $x^{-}$and $x^{+}$.

To satisfy item 3), we introduce the vertical total variation into the cost functional. If the point $x^{-} \in \mathcal{L}_{-}$is matched to point $x^{+} \in \mathcal{L}_{+}$, and $x_{y}$ is the coordinate of the intersection of the segment from $\left(x^{-}, y-1\right)$ to $\left(x^{+}, y+1\right)$ with line $y$, then we define the quantity

$$
V_{y}\left(x^{-}, x^{+}\right)=\frac{1}{2} \sum_{\Delta=+1,-1}\left|I\left(x_{y}, y+\Delta\right)-I\left(x_{y}, y\right)\right|
$$

where $I\left(x_{y}, y\right)$ is obtained by interpolating the values of $I\left(x^{-}, y-1\right)$ and $I\left(x^{+}, y+1\right)$. Notice that the values $I\left(x_{y}, y \pm 1\right)$ have to be interpolated from the values of $I$ in the lines $\mathcal{L}_{+}, \mathcal{L}_{-}$, respectively.

We define the cost $C\left(x^{-}, x^{+}\right)$as

$$
C\left(x^{-}, x^{+}\right)=\mu V_{y}\left(x^{-}, x^{+}\right)+(1-\mu) C^{1}\left(x^{-}, x^{+}\right)
$$




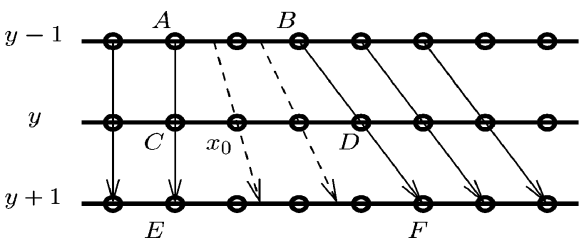

Fig. 9. Point $\left(x_{0}, y\right)$ is obtained by bilinear interpolation using the vertices of the trapezoid determined by unique correspondences.

(where $0<\mu<1$ ) if $\varphi\left(x^{-}\right)=x^{+}$and $x^{-}$is the unique point that maps to $x^{+}$, and as

$$
C\left(x^{-}, x^{+}\right)=\theta>0
$$

if either $\varphi\left(x^{-}\right)$or $\varphi^{-1}\left(x^{+}\right)$is not reduced to a point. In other words, we pay a constant amount if there is a vertical wall or a flat interval in the graph of $\varphi$ (in the case of computing correspondences in pairs of stereo images, this would amount to a occlusion or disocclusion between both lines).

Let us describe the interpolation process used to reconstruct the intermediate line $\mathcal{L}_{0}$ once we know the optimal $\varphi$. Given $\varphi$ we compute the segments determined by the unique correspondences, that is, segments determined by $\left(x^{-}, y-1\right)$ and $\left(x^{+}, y+1\right)$ when $x^{-}$is the only point mapped to $x^{+}$by $\varphi$. Each $\left(x_{0}, y\right) \in \mathcal{L}_{0}$ is contained in a trapezoid determined by points with unique correspondences (see Fig. 9) and the value of $I$ at $\left(x_{0}, y\right)$ is obtained by bilinear interpolation of the values of $I$ at the vertices of the trapezoid (points $A, B, E, F$ in Fig. 9).

A similar approach for spatial interpolation in deinterlacing has been proposed in [2]. The authors propose a hypothesis testing approach in which they want to discriminate between the dependence of the sampled lines versus its independent sampling origin. Assuming a Markovian model for the distribution of pixel values on each line, the authors propose a criterion which permits to discriminate between both hypothesis and to find the associated nondecreasing warping. The proposed criterion also takes into account the similarity between pixels in $\mathcal{L}_{-}$and $\mathcal{L}_{+}$(measured as the sum on a neighborhood of absolute values of difference between horizontal derivatives on the two lines plus the modulus of the difference of the sums of gray levels) and has a (negative) contribution of the horizontal total variation on the neighborhoods of correspondent pixels.

We have described a method for intraframe interpolation based on the interpolation of the image gray levels along the directions determined by corresponding pixels in two consecutive horizontal lines. The correspondence map is a nondecreasing warping obtained by minimizing a cost functional using a dynamic programming algorithm. These ideas will be adapted in next section for interframe interpolation.

\section{Inpainting-Based Method for Time Interpolation}

For each $j, m$, we denote by $\mathcal{L}_{j}^{m}$ the line $j$ of field $m$. Assume that we want to reconstruct line $y$ in field $n$. If we want to compute the value of the image at the point $(x, y)$ in field $n$ and there is no motion between the same point in the previous and next field, i.e., between $(x, y, n-1)$ and $(x, y, n+1)$, then

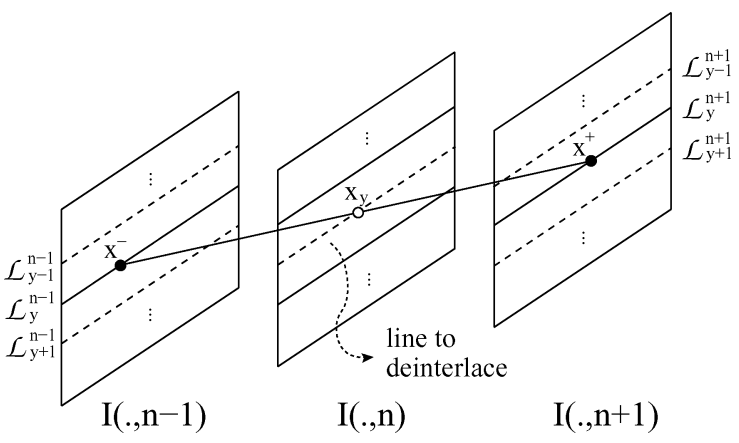

Fig. 10. We compute the warping of the lines $\mathcal{L}_{y}^{n-1}$ and $\mathcal{L}_{y}^{n+1}$ and interpolate the values of $I$ in $\mathcal{L}_{y}^{n}$ along the computed correspondences.

the best strategy would be to take an average of $I(x, y, n-1)$ and $I(x, y, n+1)$. On the contrary, this is a bad strategy when motion is present, when it is better to use a spatial interpolation, or a motion-compensated interpolation. To be able to combine both strategies we would need to use a motion detector. Instead, we have adapted the intraframe warping strategy described in Section IV-B to the case of two consecutive horizontal lines in fields $n-1$ and $n+1$, that is, of lines $\mathcal{L}_{y}^{n-1}$ and $\mathcal{L}_{y}^{n+1}$ (see Fig. 10). Using the line $\mathcal{L}_{y}^{n-1}$ in place of $\mathcal{L}_{-}$and the line $\mathcal{L}_{y}^{n+1}$ in place of $\mathcal{L}_{+}$, and using the algorithm described in Section IV-B, we compute the optimal nondecreasing warping $\varphi_{y}^{n}$ between lines $\mathcal{L}_{y}^{n-1}$ and $\mathcal{L}_{y}^{n+1}$ by minimizing the cost function (7), where $x^{-} \in \mathcal{L}_{y}^{n-1}, x^{+} \in \mathcal{L}_{y}^{n+1}, C\left(x^{-}, x^{+}\right)$is defined as in (10) and (11). The only thing to be noticed is that $C_{\text {sim }}\left(x^{-}, x^{+}\right)$ has to be replaced by the analogous function measuring the similarity of two points $x^{-}$in $\mathcal{L}_{y}^{n-1}$ and $x^{+}$in $\mathcal{L}_{y}^{n+1}$

$$
\sum_{\mathbf{h} \in \mathcal{N}^{*}(\mathbf{0})} w(\mathbf{h})\left|I\left(\mathbf{p}^{-}+\mathbf{h}, n-1\right)-I\left(\mathbf{p}^{+}+\mathbf{h}, n+1\right)\right|
$$

where $\mathbf{p}^{-}=\left(x^{-}, y\right), \mathbf{p}^{+}=\left(x^{+}, y\right)$ and the quantity $V_{y}\left(x^{-}, x^{+}\right)$is replaced by the analogous quantity measuring the total variation along the time direction

$$
\frac{1}{2} \sum_{\Delta=+1,-1}\left|I\left(x_{y}, y+\Delta, n\right)-I\left(x_{y}, y, n\right)\right|
$$

where $x_{y}$ is the coordinate of the intersection of the segment from $\left(x^{-}, y, n-1\right)$ to $\left(x^{+}, y, n+1\right)$ with line $\mathcal{L}_{y}^{n}$. The value $I\left(x_{y}, y, n\right)$ is obtained by linear interpolation using the values $I\left(x^{-}, y, n-1\right)$ and $I\left(x^{+}, y, n+1\right)$. The values $I\left(x_{y}, y \pm 1, n\right)$ are (linearly) interpolated from the values of $I$ in the lines $\mathcal{L}_{y-1}^{n}$, $\mathcal{L}_{y+1}^{n}$, respectively.

Knowing $\varphi_{y}^{n}$, line $\mathcal{L}_{y}^{n}$ is reconstructed by interpolating lines $\mathcal{L}_{y}^{n-1}$ and $\mathcal{L}_{y}^{n+1}$ using the scheme described in Section IV-B.

A related approach to this step can be found in [33] where the author proposed a dynamic programming method to compute the motion estimation using the two neighboring lines of the current one in the previous field.

\section{Combining Space and Time Interpolation}

Given an interlaced image sequence $I(\mathbf{p}, n)$, we apply to it the spatial and time interpolation methods described in Sections IV-B and IV-C, and we obtain, respectively, the progressive sequences $I_{s}(\mathbf{p}, n), I_{\tau}(\mathbf{p}, n)$. We want to combine both results using the ideas proposed in [21]. In both cases, we 
minimize the same functional, applied to two consecutive lines of the same frame in case of spatial interpolation and to the lines $\mathcal{L}_{y}^{n-1}$ and $\mathcal{L}_{y}^{n+1}$ in the case of time interpolation. Let us denote the cost $C$ defined by (10) and (11) by $C_{s}$ in case of the spatial interpolation and by $C_{\tau}$ in the case of time interpolation.

Now, let us consider a line $\mathcal{L}_{y}^{n}$ which was missing in the original interlaced sequence and has been reconstructed in both images $I_{s}(\mathbf{p}, n), I_{\tau}(\mathbf{p}, n)$. As described in Section III, we can combine both results $I_{s}(\mathbf{p}, n), I_{\tau}(\mathbf{p}, n)$ by linear combination with coefficients which are functions of a reliability measure computed for each of them. We define the reliability as a decreasing function of the cost, so that lower cost implies higher reliability, concretely, we use

$$
\epsilon_{s}(\mathbf{p}, n)=e^{-C_{s}(\mathbf{p}, n)} \quad \epsilon_{\tau}(\mathbf{p}, n)=e^{-C_{\tau}(\mathbf{p}, n)}
$$

where $(\mathbf{p}, n)$ is a point on the line $\mathcal{L}_{y}^{n}$. Then we combine $I_{s}(\mathbf{p}, n), I_{\tau}(\mathbf{p}, n)$ using the formula

$$
\begin{aligned}
& I_{s \tau}(\mathbf{p}, n)=\frac{\epsilon_{s}(\mathbf{p}, n)}{\epsilon_{s}(\mathbf{p}, n)+\epsilon_{\tau}(\mathbf{p}, n)} I_{s}(\mathbf{p}, n) \\
& +\frac{\epsilon_{\tau}(\mathbf{p}, n)}{\epsilon_{s}(\mathbf{p}, n)+\epsilon_{\tau}(\mathbf{p}, n)} I_{\tau}(\mathbf{p}, n) .
\end{aligned}
$$

We have also experimented with another choice of weights. When computing the coefficients $\epsilon$, instead of considering just the value of the cost $\left(C_{s}\right.$ or $\left.C_{\tau}\right)$ at pixel $\mathrm{p}$, we average the cost on a neighborhood of $\mathbf{p}$, so we get

$$
\begin{aligned}
& \bar{C}_{s}(\mathbf{p}, n)=\sum_{\Delta=-d}^{\Delta=+d} C_{s}(\mathbf{p}+(\Delta, 0), n) \\
& \bar{C}_{\tau}(\mathbf{p}, n)=\sum_{\Delta=-d}^{\Delta=+d} C_{\tau}(\mathbf{p}+(\Delta, 0), n)
\end{aligned}
$$

where $d$ is a positive integer. If $\bar{C}_{s}<\bar{C}_{\tau}$, our weights $\epsilon$ are defined as

$$
\epsilon_{s}(\mathbf{p}, n)=1, \quad \epsilon_{\tau}(\mathbf{p}, n)=e^{-\sigma\left|\bar{C}_{\tau}(\mathbf{p}, n)-\bar{C}_{s}(\mathbf{p}, n)\right|}
$$

and if $\bar{C}_{\tau}<\bar{C}_{s}$, they are defined as

$$
\epsilon_{\tau}(\mathbf{p}, n)=1, \quad \epsilon_{s}(\mathbf{p}, n)=e^{-\sigma\left|\bar{C}_{\tau}(\mathbf{p}, n)-\bar{C}_{s}(\mathbf{p}, n)\right|}
$$

where $\sigma>0$. Experimentally, the deinterlacing results obtained using the weights defined in (12) or those in (14) and (15) are almost identical in terms of mean square error (MSE), though we have observed that the second choice of coefficients may yield less visual artifacts in some video sequences.

These formulas are similar in spirit to those used in [21], though different in form. They are similar to those used in [38], where the authors observed that $I_{s \tau}(\mathbf{p}, n)$ is the value of $\mathcal{I}$ that minimizes the quantity

$$
\epsilon_{s}(\mathbf{p}, n)\left(\mathcal{I}-I_{s}(\mathbf{p}, n)\right)^{2}+\epsilon_{\tau}(\mathbf{p}, n)\left(\mathcal{I}-I_{\tau}(\mathbf{p}, n)\right)^{2} .
$$

Let us comment on a technical point. Notice that the coefficients $\epsilon_{s}(\mathbf{p}, n), \epsilon_{\tau}(\mathbf{p}, n)$ in (12) and (13) are defined on points $(\mathbf{p}, n)$ on the line $\mathcal{L}_{y}^{n}$. However, originally, the costs $C_{s}(\mathbf{p}, n)$,
$C_{\tau}(\mathbf{p}, n)$ were not defined on those points. Let us explain how to compute $C_{s}$ on points $(\mathbf{p}, n)$ in $\mathcal{L}_{y}^{n}$, the case of $C_{\tau}$ being analogous. With that purpose, let $\varphi$ be the optimal nondecreasing warping between $\mathcal{L}_{y-1}^{n}$ and $\mathcal{L}_{y+1}^{n}$ obtained for the space (intraframe) interpolation of $\mathcal{L}_{y}^{n}$. As explained in Section IV-B, recall that each point $\mathbf{p}=\left(x_{0}, y\right) \in \mathcal{L}_{y}^{n}$ is contained in a trapezoid determined by points with unique correspondences (see Fig. 9). Referring to Fig. 9, point $\left(x_{0}, y\right)$ is contained in the trapezoid determined by the correspondences $\varphi(A)=E$ and $\varphi(B)=F$. Since $\left(x_{0}, y\right)$ is in the segment determined by $C$ and $D$, we have that $\left(x_{0}, y\right)=\rho C+(1-\rho) D$ for some $\rho \in[0,1]$. Then we define the cost $C_{s}(\mathbf{p}, n)$ by linear interpolation of the costs of the correspondences $A$ to $E$ and $B$ to $F$, that is, $C_{s}(\mathbf{p}, n)=\rho C_{s}(A, E)+(1-\rho) C_{s}(B, F)$. In a similar way, we define $C_{\tau}(\mathbf{p}, n)$.

\section{EXPERIMENTAL RESULTS}

In this section, we will discuss the results that are obtained with the proposed method.

Our dynamic programming implementation is inspired by the one proposed by Cox et al. for stereo matching [10]. It is written in $\mathrm{C}++$ under Linux running on a Dual-core 3-GHz 2-Gb PC. The per-frame complexity is $O(S)$ where $S=M \times N$ is the number of image pixels. For a frame of TV dimensions $(640 \times$ 480 ) the total processing time per frame is roughly $1.3 \mathrm{~s}$. This may seem rather too much time given the low complexity of the algorithm, but the bottleneck lies not in the CPU processing time but rather in the data-bus transfer rate. Please bear in mind that for each $M \times 1$ line in each frame field we have to create two $M \times M$ floating-point matrices (for $C_{s}$ and $C_{\tau}$ ) and two unsigned char matrices (for backtracking in computing the optimal path,) which in the case of TV resolution and using 4 bytes per floating-point number, imply the need to transfer between memory and the CPU an amount of $5 \times 480 \times 640 \times 640 \times 2=$ $1.83 \mathrm{~GB}$ per frame, while typical data-bus transfer rates range from 1.5 to $8 \mathrm{~GB} / \mathrm{s}$. Of course, if for each matrix, we only compute a diagonal band, the speed increase is considerable, but we are still far from deinterlacing at a rate of 25-30 frames per second. We are currently exploring GPU acceleration in order to bring our algorithm down to real time: see, for instance, [18] and [24], where they use programmable graphics hardware to solve problems which in essence are quite similar to IPC.

All of our tests have been executed using the same set of parameters. $\mathcal{N}(\mathbf{0})$ is a neighborhood of $5 \times 3$ pixels, $w(\mathbf{h})=$ $1 / \operatorname{card}\left(\mathcal{N}^{*}(\mathbf{0})\right)$ where $\operatorname{card}(\mathbf{A})$ is the cardinality of the set $\mathbf{A}$ and $\mathcal{N}^{*}(\mathbf{0})$ is the intersection of $\mathcal{N}(\mathbf{0})$ with the known lines. In our case, $\operatorname{card}\left(\mathcal{N}^{*}(\mathbf{0})\right)=9$. We do not observe significant differences in the results if we vary the size of $\mathcal{N}(\mathbf{0})$ between $5 \times 3$ and $9 \times 5$. For $\alpha, \beta$ we have used $\alpha=0.9, \beta=0.1$. Actually, given the way these parameters appear in the computation of the cost matrix [see (9)], it is only their ratio what we should take into account, i.e., we may consider as a parameter just $\alpha / \beta$. We have tested several values of this ratio, in the range 5.7-19.0, with no significant change in the MSE of the results. For $\mu$ [see (10)], we have chosen $\mu=0.5$. We have observed that if we decrease the value of this parameter the results remain almost unchanged when we use the choice of weights presented 

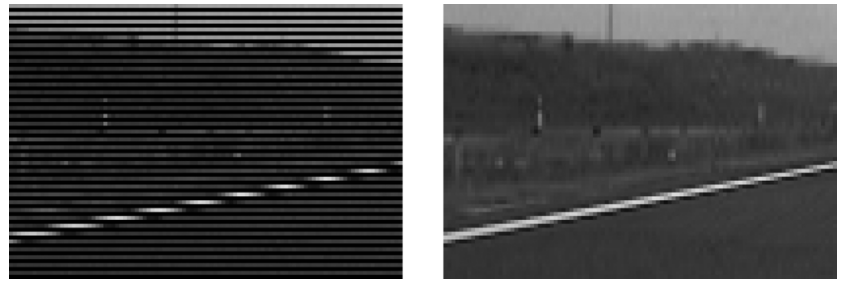

Fig. 11. Example of IPC applying the geometrical-based approach. Left: Interlaced image. Right: IPC reconstructed image obtained with our proposed spatial reconstruction approach.

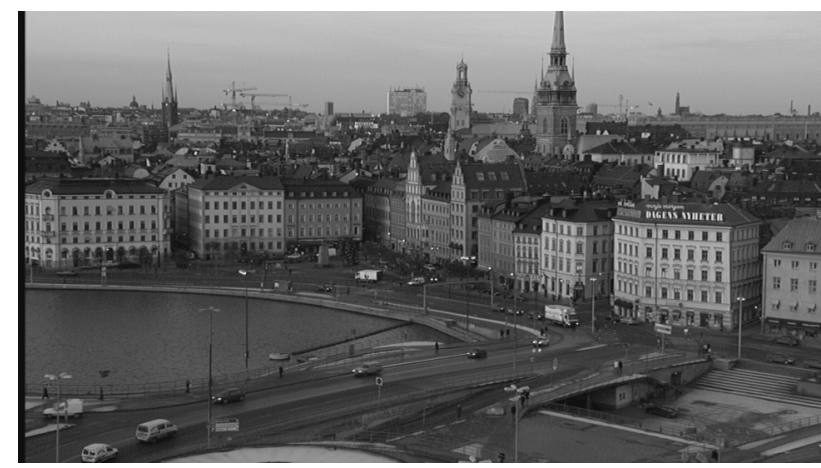

Fig. 12. Frame of the original progressive Stockholm sequence. We have applied our spatial, temporal, and combined spatiotemporal (STIM) method to the interlaced Stockholm sequence. The MSEs between the original progressive material and the result of our spatial, temporal and combined spatiotemporal interpolation methods are MSE $=31.76,10.34,9.43$, respectively. Some details of the results are displayed in Figs. 13 and 14.

above in (14) and (15). Recapping, the algorithm is quite robust, in terms of MSE, to changes in these parameters. ${ }^{3}$

Let us first see an example that shows how our algorithm works. For that purpose, let us consider a progressive image of the Highway sequence. This image is first artificially interlaced, as shown in Fig. 11, left. Then, on the interlaced image, our proposed spatial reconstruction approach is applied. The IPC reconstruction result is shown in Fig. 11, right. As it can be seen, our method has been able to properly reconstruct the white slanted line of the highway as well as both black slanted lines at both sides of the white one. Other classical methods such as the DOI would fail to properly reconstruct the geometrical structure at all pixels along the slanted line due to their local processing technique (see Fig. 18, top row).

In Fig. 12, we display a frame of the original progressive Stockholm sequence. This sequence shows a slow panning view over the old town of Stockholm. It contains many buildings and windows which have a periodic structure. We have applied the different steps of our algorithm to the full Stockholm sequence. We shall display some details of the results in Figs. 13 and 14. Let us mention that the mean squared error between the original progressive material and the result of our spatial inpainting is MSE $=31.76$, with the temporal inpainting we get MSE $=10.34$, and with the combined spatiotemporal interpolation

${ }^{3}$ There are other two numbers that have to be set: one is $\theta$ [see (11)], which we have taken directly from [10], and the other is the size of the diagonal band, which is essential for optimization (for this number we have chosen as a value one-tenth of the total number of columns).
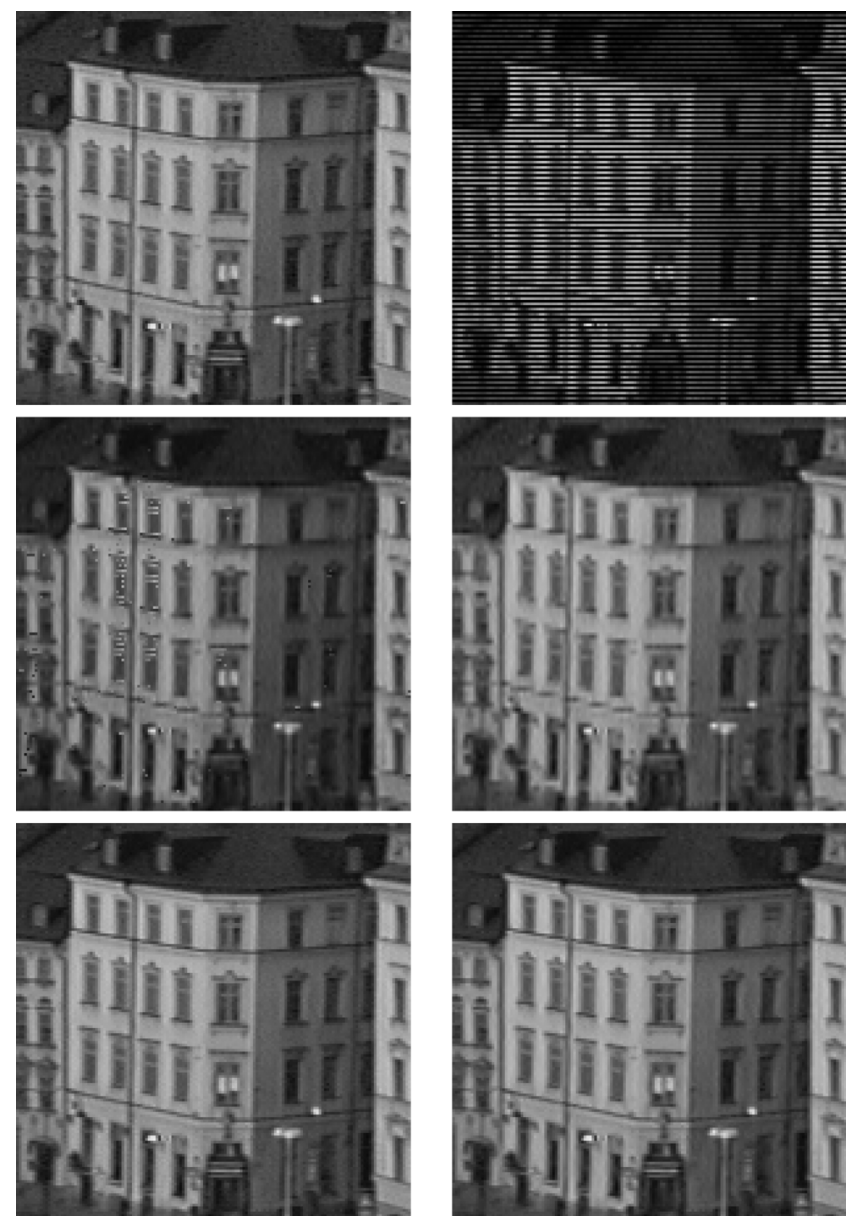

Fig. 13. Example of performance comparison of DOI with our inpainting-based spatial, temporal, and combined STIMs. Top, left: Detail of a frame of the original progressive Stockholm sequence. Top, right: Corresponding interlaced detail. Middle, left: Deinterlaced result obtained with DOI. Middle, right: Result obtained with our spatial interpolation step. Bottom, left: Deinterlaced result obtained with our temporal interpolation step. Bottom, right: Result obtained with the combined STIM.

the error is MSE $=9.43$. We have also implemented the dynamic time warping scheme in [2], and in this case we obtained an MSE of 35.77 .

In Fig. 13, we compare the performance of the directional oriented interpolator (DOI) with our inpainting-based spatial interpolation approach and with the other steps of our approach. The DOI method performs well in general but fails with periodic structures. To show this, in Fig. 13(a), we display a detail of Fig. 12 corresponding to the Stockholm sequence. The corresponding interlaced detail is shown in Fig. 13(b). The output of the DOI deinterlaced result is shown in Fig. 13(c). As it can be seen, many artifacts appear in the reconstructed image. This is due to the local interpolation decision made by the approach. In Fig. 13(d), we show the reconstructed image obtained using our spatial interpolation approach. As it can be seen, the algorithm has properly reconstructed most of the geometric structures present in the image, but some details could not be properly reconstructed. See, for instance, the long black slanted lines below the windows. One may observe segments of these long lines in the interlaced image. However, these segments have not been connected in the spatially interpolated image since they are 

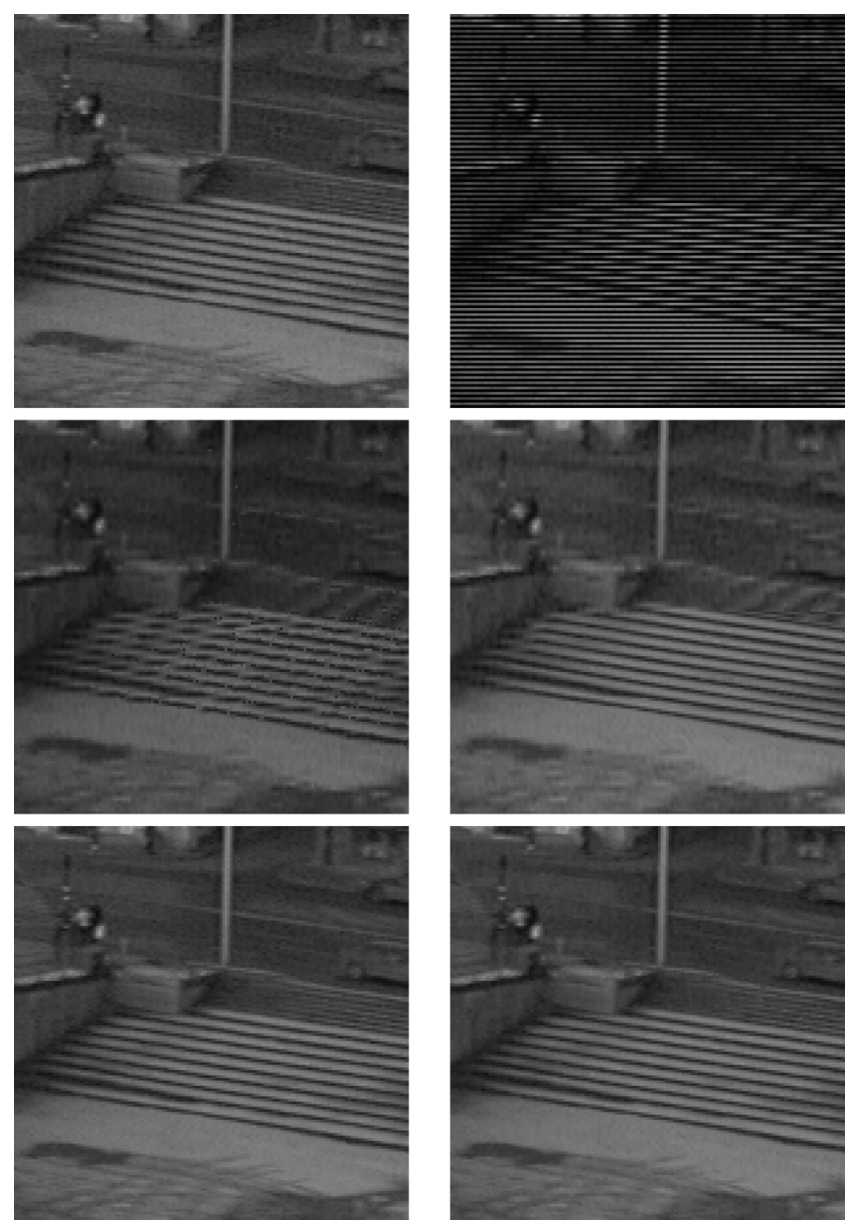

Fig. 14. We display a second detail of a frame of the Stockholm sequence. From top to bottom and left to right, we display (for the sake of comparison) the detail of the original frame, the corresponding interlaced detail, and the results obtained with DOI, and our spatial, temporal, and combined STIM results.

too far away. In other words, it is "cheaper" (in cost terms) to keep them as separated segments. In Fig. 13(e), we display the result obtained with our temporal inpainting interpolation step. The result of the proposed STIM is shown in Fig. 13(f). As can be seen, the combined approach is able to properly reconstruct most details present in the image.

In Fig. 14, we display a second detail of the Stockholm sequence, see Fig. 12. We display the detail in the original progressive sequence (for the sake of comparison), the corresponding interlaced image, and the deinterlaced results obtained with DOI, our spatial, temporal, and combined STIM methods, respectively. We see that the result of our spatial interpolation is better than DOI, the temporal interpolation is better than the spatial, and the combined result is visually comparable with the temporal one, though it is better in terms of MSE as can be seen in Fig. 12.

Our method has also been tested against the following methods (see Section II): line average (LAV), field average (FAV), directional oriented interpolation (DOI), spatiotemporal ELA (STELA), four field motion adaptive (4FMA, it uses LAV in the presence of motion and field weaving if no motion is present), motion compensated (based on the motion estimation proposed in [6] described in next paragraph), and adaptive

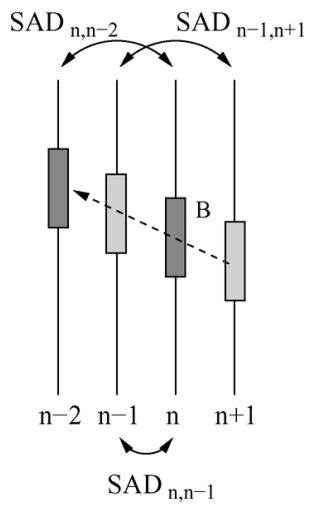

Fig. 15. Four-field motion estimation scheme.

recursive (AR) motion compensated (the method proposed in [12]; see Section II-D).

For the MC and AR schemes a motion estimation technique is needed. In this paper, a four-field block-based motion estimation technique similar to the one presented in [6] has been implemented. The scheme is depicted in Fig. 15. Let $I(\mathbf{p}, n)$ denote the field at point $\mathbf{p}$ and time instant $n$. The motion vector $\mathbf{v}$ of a pixel $\mathbf{p}$ is estimated based on assessing three values of a sum of absolute differences (SAD), one between pixels of a block $B$ in fields $n$ and $n-2, \operatorname{SAD}_{n, n-2}(B, \mathbf{p}, \mathbf{v}, n)=\sum_{\mathbf{h} \in B} \mid I(\mathbf{p}+$ $\mathbf{h}, n)-I(\mathbf{p}+\mathbf{h}-2 \mathbf{v}, n-2) \mid$, a second between fields $n-1$ and $n+1, \operatorname{SAD}_{n-1, n+1}(B, \mathbf{p}, \mathbf{v}, n)=\sum_{\mathbf{h} \in B} \mid I(\mathbf{p}+\mathbf{h}+\mathbf{v}, n+$ 1) $-I(\mathbf{p}+\mathbf{h}-\mathbf{v}, n-1) \mid$, and a third using fields $n$ and $n-1$, $\mathrm{SAD}_{n, n-1}(B, \mathbf{p}, \mathbf{v}, n)=\sum_{\mathbf{h} \in B} \mid I(\mathbf{p}+\mathbf{h}, n)-I(\mathbf{p}+\mathbf{h}-\mathbf{v}, n-$ 1)|.

Motion vector $\hat{\mathbf{v}}$ is estimated minimizing the following term:

$$
\hat{\mathbf{v}}=\frac{1}{2}\left[\arg \min _{\mathbf{v} \in \mathrm{SW}}\left(\mathrm{SAD}_{n, n-2}+\mathrm{SAD}_{n-1, n+1}+\mathrm{SAD}_{n, n-1}\right)\right]
$$

where SW is the search window, and the arguments of SAD has been dropped to simplify notation. Note that the terms $\mathrm{SAD}_{n, n-2}$ and $\mathrm{SAD}_{n-1, n+1}$ compare blocks of fields with the same parity while the fields involved in $\mathrm{SAD}_{n, n-1}$ are of different parity. The former terms are common in interlaced-based motion estimation and detection [9], whereas the latter is used to analyze the presence of fast motion, for instance in [7].

We have implemented the previous scheme using a block $B$ of size $21 \times 21$ pixels for high resolution sequences, and of $8 \times 8$ pixels for low resolution ones. Motion between fields of the same parity is estimated using integer pixel precision. Thus, between two successive fields the motion vectors have precision of 0.5 . The vector given in (16) is found by exploring a search window SW of size $51 \times 21$. Note that the vertical component of the search window is approximately one half of the horizontal one since motion estimation is performed on the fields. Moreover, we use the hierarchical search method described in [35].

In Table I, we compare the complexity of all these algorithms. The complexity is expressed in number of operations per pixel to deinterlace, and we have computed the figures both for memory access operations (MAO) and floating point operations (FPO). For instance, for the LAV deinterlacer, the output is computed with (1): in order to deinterlace each pixel, 
TABLE I

MAO (MEMORY ACCESS OPERATIONS) AND FPO (FLOATING POINT OPERATIONS) PER PIXEL, FOR SEVERAL DEINTERLACING METHOdS. SEE TEXT For DetaILS

\begin{tabular}{|r|l|l|}
\hline Method & $M A O$ & FPO \\
\hline LAV & 3 & 2 \\
FAV & 3 & 2 \\
4FMA & 7 & 7 \\
ELA & 7 & 8 \\
STELA & 13 & 26 \\
DOI & 387 & 386 \\
STIM (D=21) & 1459 & 1862 \\
STIM (D=41) & 2819 & 3582 \\
MC (L=3) & 2370 & 3557 \\
MC (L=1) & 6425 & 9642 \\
\hline
\end{tabular}

a sum and a division are performed (requiring two FPO) and two pixel accesses are needed (requiring two MAO), plus one $\mathrm{MAO}$ in order to store the result in the corresponding pixel position (3 MAO in total). Four our proposed STIM algorithm we show the figures for two different choices of the width $D$ of the diagonal band used in the computation of the cost matrix: $D=21,41$. For the MC scheme we show the figures for two choices of a multiscale implementation: just one level $(L=1)$, and three levels $(L=3)$. We must stress that the MC technique is block-based, with blocks of size $21 \times 21$ pixels, i.e., we compute one vector per $21 \times 21$ block, whereas in STIM matches are computed per-pixel and not per-block. Despite this fact, STIM (with $D=21$ ) needs roughly one fifth of the operations required by the one-level implementation of the MC scheme, and has approximately half the complexity of the three-level implementation of the MC scheme.

Several different test sequences have been employed, three of them are of CIF dimensions $(352 \times 288)$ while the other four are of high resolution $(1280 \times 720)$. In Fig. 18(a), the key frame of each sequence is shown on the left column. We include a brief description of them here.

- Mobile $(352 \times 288)$ : The Mobile \& Calendar sequence. The camera pans left following the movement of an electric train, with a calendar moving vertically behind it. In the background there is a wallpaper with several figures. Frames 0 to 29 have been used.

- Paris $(352 \times 288)$ : A man and a woman moving in front of a detailed static background. Frames 0 to 29 have been used.

- Highway $(352 \times 288)$ : View from inside a car traveling along a highway. Features some fast moving objects and slanted lines with different slopes. Used frames 0 to 29.

- Stockholm $(1280 \times 720)$ : Slow pan over a city landscape. Lots of detail and structue. Frames 100 to 129 have been used for the tests.

- Parkrun $(1280 \times 720)$ : Slow lateral dolly of a man running in a park. Many details and different textures. Used frames 20 to 49.

- Train $(1280 \times 720)$ : A calendar with text moves vertically, a toy train moves horizontally. Different textures and printed figures. Used frames 460 to 489.

- Shields $(1280 \times 720)$ : A man points to a wall with different shields. Used frames 440 to 469 since it contains a slow zoom-in.

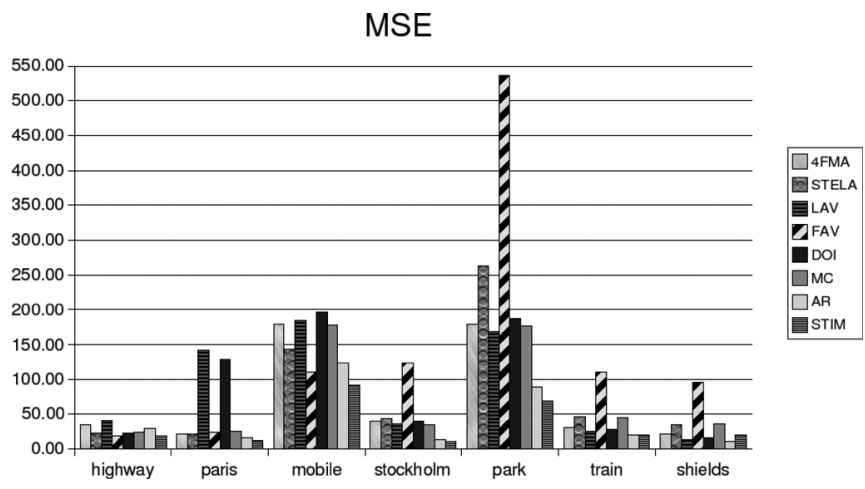

Fig. 16. Comparison of mean MSE obtained for different sequences (see text). For each sequence, and from left to right, the following methods are shown: 4FMA, STELA, LAV, FAV, DOI, MC, AR, and STIM.

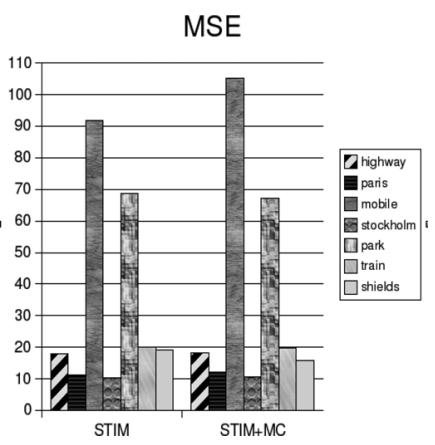

Fig. 17. Comparison of mean MSE obtained with regular (left) STIM, and with STIM combined with (right) MC.

Note that the selected testbed contains sequences of different sizes and different nature. Some of them include many details, such as the Parkrun or the Stockholm sequences. The sequences include also different types of motion, something necessary in order to test the performance of our approach.

It should also be noted that the previous sequences are in progressive format. That is, the full resolution image is available, so we can always compute the MSE between the progressive original and the deinterlaced results. For testing purposes, each image of the sequence is interlaced and then reconstructed.

Fig. 16 compares the performance of the IPC methods over the different sequences. In particular, for each sequence seven different methods have been tested: 4FMA, STELA, LAV, FAV, DOI, MC, AR, and STIM. The latter corresponds to our method (spatiotemporal inpainting method). The mean squared error between the original progressive and the reconstructed IPC image can be considered an objective measure of the performance. In Fig. 16, the mean of the mean squared error over the selected images from each sequence has been computed. As it can be seen, the proposed STIM method performs better than the other approaches in most cases. Indeed, only the MC and AR approaches are comparable to our approach. In our technique, however, we do not make use of motion estimation to deinterlace.

Moreover, we observe also in Fig. 16 that LAV and FAV perform differently depending on the content of the sequence. For instance, FAV does not perform well in sequences that have a global transformation such as a panning or zoom (see the MSE data for the sequences Stockholm, Park, Train, and Shields). On 


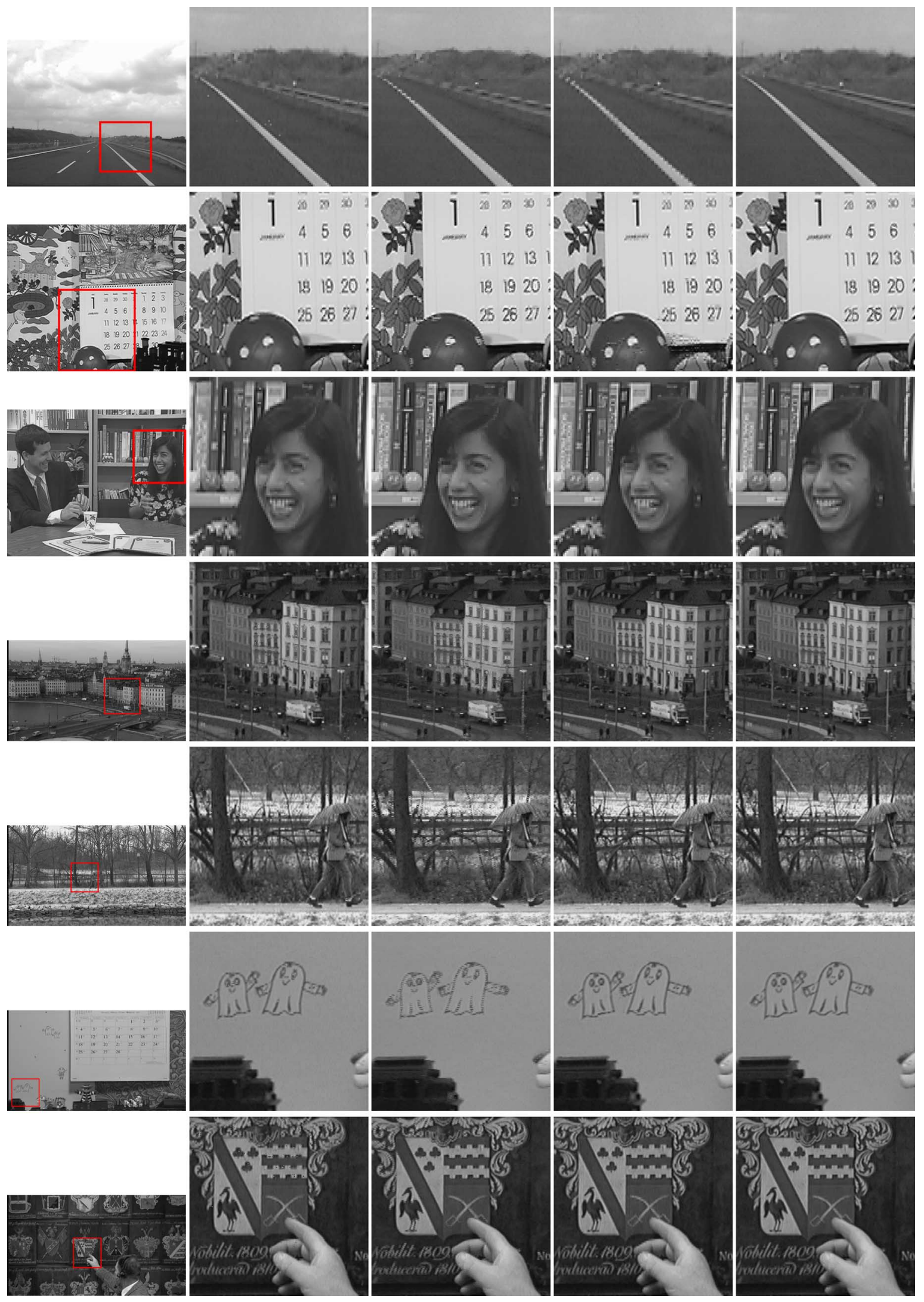

Fig. 18. Comparison of several IPC methods. First column: original frame. Columns 2 to 4: details of results with DOI, STELA, and AR. Last column: our result. The sequences shown are, from top top bottom: Highway, Paris, Mobile, Stockholm, Parkrun, Train, Shields. See text for details. Please watch the videos available at http://www.tecn.upf.es/ garrido/deinterlace.

the other hand, LAV does not perform well on static zones (see the MSE data for the Paris sequence). Thus, a motion adaptive scheme (in our case 4FMA) may combine the strength of both approaches to obtain a lower MSE, see graph. STELA and DOI 

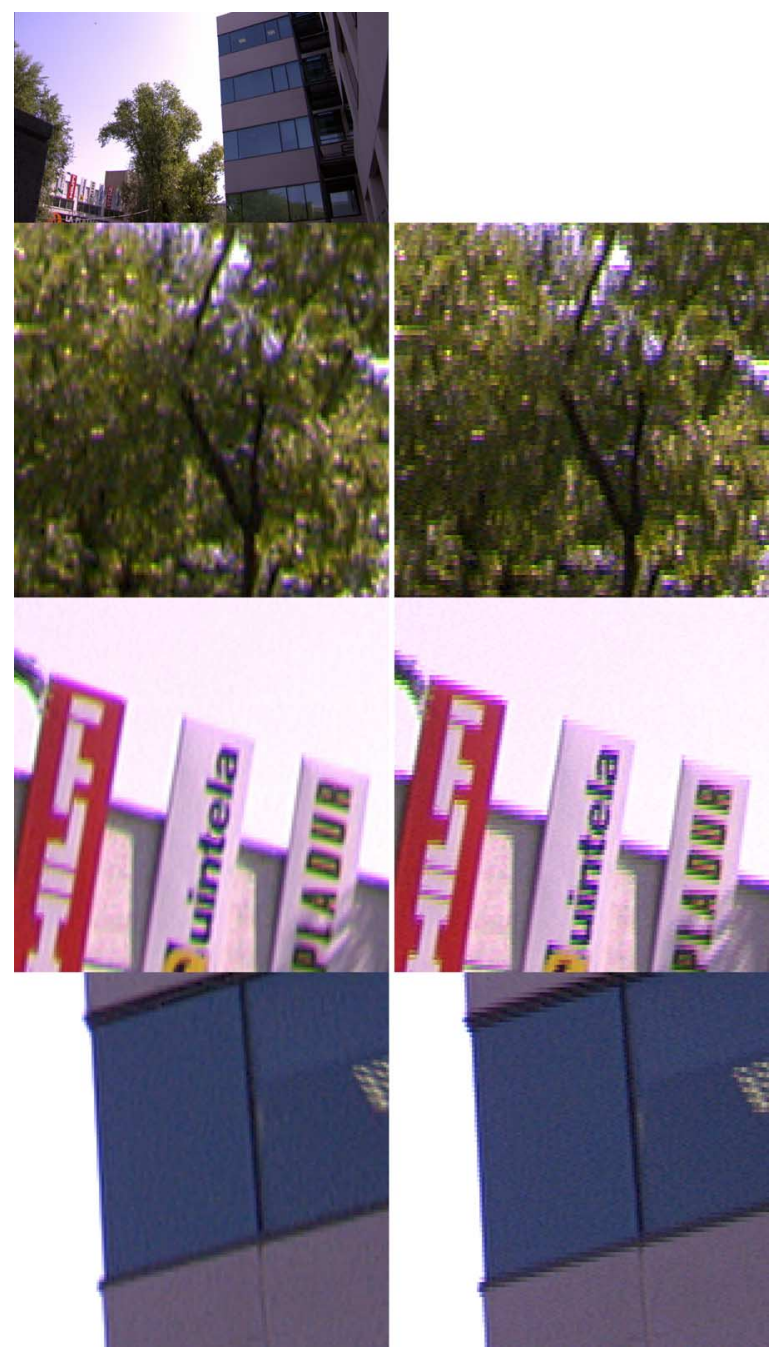

Fig. 19. (Top) One frame and (left column) details of the deinterlacing results obtained with STIM on a high definition, originally interlaced sequence with a substantial vertical motion (a camera pan-up.) The right column shows the original interlaced sequence in a field weaving fashion: two fields in the same frame. Video available at http://www.tecn.upf.es/ garrido/deinterlace.

perform, in general, considerably well, but they introduce many artifacts in the reconstructed sequence which are particularly annoying if the sequence is played.

Even if the MSE is a widely used performance measure, it is known that the MSE it not enough to measure the subjective quality of the reconstructed image [13]. In the case of IPC, several measures other than MSE may be used for assessing the performance of a particular approach [3]. Indeed, in the IPC case, even if no artifacts appear in a single image, many artifacts may appear when the sequence is played. In our case, visual inspection shows that the results obtained with STIM suffer from less artifacts than the results obtained with the MC and AR approaches. Please watch the videos available at http://www.tecn. upf.es/ garrido/deinterlace. For instance, in highly textured sequences, such as Stockholm or Parkrun, an incorrect estimation of the motion field produces very noticeable artifacts in the reconstruction with MC or AR. We do not have these problems since we do not make use of motion estimation. Furthermore, if we refine our results by combining our approach with a MC procedure, the quality of the results does not improve noticeably, and in some cases it even decreases; see Fig. 17.

In Fig. 18, a comparison between several IPC methods is shown. In the first column, the original progressive image is shown. Columns 2 to 4 show a detail of the reconstructed image using DOI, STELA and AR, respectively. The last column shows the result obtained by the STIM method. We can observe that the perfomance of the proposed method is quite consistent, while for the other approaches the performance varies noticeably depending on the image content. These videos are at http://www.tecn.upf.es/ garrido/deinterlace.

Finally, Fig. 19 shows one frame and details of the deinterlacing results obtained with STIM on a high definition, originally interlaced sequence with a substantial vertical motion (a camera pan-up.) Since this sequence is originally interlaced we can not compute the MSE to measure the quality of the results, but the details highlighted in the figure show that boundaries, banners, textured regions are all reconstructed properly, and the video presents no visual artifacts.

\section{CONCLUSION}

We have proposed in this paper an inpainting-based method for interlaced to progressive video conversion. An inpainting technique which fills in the missing lines by reconstructing the image level sets (subject to an ordering constraint on the level lines) is applied both to the spatial and the temporal domains, and the results are combined via a weighted average. A dynamic programming procedure is used, and the total complexity of the procedure is $O(S)$, where $S$ is the number of pixels in the image. The results are comparable or better than those obtained with state of the art, motion-compensated IPC methods, but at a much lower computational cost since we do not need to perform motion field estimation. We are currently investigating on a GPU implementation of our algorithm in order to achieve real-time, high-quality IPC with a regular PC.

\section{ACKNOWLEDGMENT}

The authors would like to thank to J. Barcons for his help and suggestions in optimizing their algorithm.

\section{REFERENCES}

[1] M. Achiha, K. Ishikura, and T. Fukinuki, "A motion-adaptive highdefinition converter for NTSC color TV signals," SMPTE J., vol. 93, pp. 470-476, 1984.

[2] A. Almog, A. Levi, and A. M. Bruckstein, "Spatial de-interlacing using dynamic time warping," in Proc. IEEE Int. Conf. Image Processing, 2005, vol. 2, pp. 1010-1013.

[3] E. B. Bellers and G. De Haan, "On video formats and coding efficiency," pp. 25-32, 2001

[4] M. Bertalmío, L. Vese, G. Sapiro, and S. Osher, "Simultaneous structure and texture image inpainting," IEEE Trans. Image Process., vol. 12, no. 8, pp. 882-889, Aug. 2003.

[5] A. M. Bock, "Motion-adaptive standards conversion between formats of similar field rates," Signal Process. Image Commun., vol. 6, pp. 275-280, 1994.

[6] Y. L. Chang, P. H. Wu, S. F. Lin, and L. G. Chen, "Four field local motion compensated de-interlacing," in Proc. IEEE Int. Conf. Acoustics, Speech, Signal Processing, 2004, vol. 5, pp. 253-256.

[7] Y.-L. Chang, C.-Y. Chen, S.-F. Lin, and L.-G. Chen, "Four field variable block size motion compensated adaptive de-interlacing," presented at the IEEE Int. Conf. Acoustics, Speech, and Signal Processing, 2005. 
[8] M. J. Chen, C. H. Huang, and C. T. Hsu, "Efficient de-interlacing technique by inter-field information," IEEE Trans. Consum. Electron., vol. 50, no. 4, pp. 1202-1208, Nov. 2004

[9] C. Ciuhu and G. de Haan, "Motion estimation on interlaced video," Proc. SPIE, vol. 5685, pp. 718-729, 2005.

[10] I. J. Cox, S. L. Hingorani, S. B. Rao, and B. M. Maggs, "A maximum likelihood stereo algorithm," Comput. Vis. Image Understand., vol. 63, no. 3, pp. 542-567, 1996.

[11] A. Criminisi, P. Perez, and K. Toyama, "Region filling and object removal by exemplar-based image inpainting," IEEE Trans. Image Process., vol. 13, no. 9, pp. 1200-1212, Sep. 2004.

[12] G. De Haan and E. B. Bellers, "De-interlacing of video data," IEEE Trans. Consum. Electron., vol. 43, no. 8, pp. 819-825, Aug. 1997.

[13] G. De Haan and E. B. Bellers, "Deinterlacing-An overview," Proc. IEEE, vol. 86, no. 9, pp. 1839-1857, Sep. 1998.

[14] T. Doyle, "Interlaced to sequential conversion for EDTV applications," in Proc. 2nd. Int. Workshop Signal Processing of HDTV, 1988, pp. $412-430$.

[15] T. Doyle and M. Looymans, "Progressive scan conversion using edge information," in Proc. Signal Processing HDTV II, 1990, pp. 711-721.

[16] A. A. Efros and T. K. Leung, "Texture synthesis by non-parametric sampling," in Proc. ICCV, 1999, vol. 2, pp. 1033-1038.

[17] P. D. Filliman, T. J. Christopher, and R. T. Keen, "Interlace to progressive scan converter for IDTV," IEEE Trans. Consum. Electron., vol. 38, no. 8, pp. 135-144, Aug. 1992.

[18] M. Gong and Y. G. Yang, "Near real-time reliable stereo matching using programmable graphics hardware," in Proc. IEEE Computer Society Conf. Computer Vision and Pattern Recognition, Jun. 2005, vol. 1, pp. 924-931.

[19] P. Haavisto, J. Juhola, and Y. Neuvo, "Scan rate-up conversion using adaptive weighted median filtering," in Proc. Signal Processing of HDTV II, 1990, pp. 703-710.

[20] Y. Kim and Y. Cho, "Motion adaptive deinterlacing algorithm based on wide vector correlations and edge dependent motion switching," in Proc. HDTV Workshop, 1995, pp. 8B9-8B16.

[21] J. Kovačević, R. J. Safranek, and E. M. Yeh, "Deinterlacing by successive approximation," IEEE Trans. Image Process., vol. 6, no. 2, pp. 339-344, Feb. 1997.

[22] C. L. Lee, S. Chang, and C. W. Jen, "Motion detection and motion adaptive pro-scan conversion," in Proc. IEEE Int. Symp. Circuits and Systems, Jun. 1991, vol. 1, pp. 666-669.

[23] M. Lee, J. Kim, J. Lee, K. Ryu, and D. Song, "A new algorithm for interlaced to progressive scan conversion based on directional correlations and its ic design," IEEE Trans. Consum. Electron., vol. 40, no. 5, pp. 119-129, May 1994.

[24] W. Liu, B. Schmidt, G. Voss, A. Schroder, and W. Muller-Wittig, "Biosequence database scanning on a gpu," in Proc. 20th Int. Parallel and Distributed Processing Symp., Apr. 2006, pp. 1-8.

[25] S. Masnou, "Filtrage et desocclusion d'images par méthodes d'ensembles de Niveau," Ph.D. dissertation, Univ. Paris Dauphine, France, 1998.

[26] S. Masnou, "Disocclusion: A variational approach using level lines," IEEE Trans. Image Process., vol. 11, no. 1, pp. 68-76, Jan. 2002.

[27] S. Masnou and J. M. Morel, "Level lines based disocclusion," in Proc. 5th IEEE Int. Conf. Image Processing, 1998, pp. 259-263.

[28] Y. Matsushita, E. Ofek, X. Tang, and H. Y. Shum, "Full-frame video stabilization," in Proc. IEEE Computer Society Conf. Computer Vision and Pattern Recognition, Jun. 2005, vol. 1, pp. 50-57.

[29] H. S. Oh, Y. Kim, Y. Jung, A. W. Morales, and S. J. Ko, "Spatiotemporal edge-based median filtering for deinterlacing," in Proc. Dig. Tech. Papers. Int. Conf. Consumer Electronics, Jun. 2000, pp. 52-53.

[30] J. Salo, Y. Neuvo, and V. Hameenaho, "Improving TV picture quality with linear-median type operations," IEEE Trans. Consum. Electron., vol. 34, no. 8, pp. 373-379, Aug. 1988.

[31] J. Salonen and S. Kalli, "Edge adaptive interpolation for scanning rate conversion," in Proc. Signal Processing of HDTV IV, 1993, pp. 757-764.

[32] R. Simonetti, S. Carrato, G. Ramponi, and A. P. Filisan, "Deinterlacing of HDTV images for multimedia applications," in Proc. Signal Processing of HDTV IV, 1993, pp. 765-772.

[33] C. Sun, "De-interlacing of video images using a shortest path technique," IEEE Trans. Consum. Electron., vol. 47, no. 5, pp. 225-230, May 2001.

[34] M. Suyigama, S. Hirahata, K. Katsumata, K. Ishikura, A. Okuda, T. Sakamoto, T. Matono, S. Suzuki, I. Nakagawa, and M. Achiha, "High quality digital TV with frame store processing," IEEE Trans. Consum. Electron., vol. 33, no. 8, pp. 98-108, Aug. 1987.
[35] A. M. Tekalp, Digital Video Processing. Englewood Cliffs, NJ: Prentice-Hall, 1995.

[36] D. Tschumperlé and B. Besserer, "High quality deinterlacing using inpainting and shutter-model directed temporal interpolation," in Proc. ICCVG, Sep. 2004, pp. 1-7.

[37] F. M. Wang, D. Anastassiou, and A. N. Netrvali, "Time recursive deinterlacing for IDTV and pyramid coding," Signal Process.: Image Commun., vol. 2, pp. 365-374, 1990.

[38] Y. Wexler, E. Schechtman, and M. Irani, "Space-time video completion," in Proc. IEEE Computer Society Conf. Computer Vision and Pattern Recognition, Jun. 2004, vol. 1, pp. 120-127.

[39] S. Yang, Y. Y. Jung, Y. H. Lee, and R. H. Park, "Motion compensation assisted motion adaptive interlaced-to-progressive conversion," IEEE Trans. Circuits Syst. Video Technol., vol. 14, no. 9, pp. 1138-1148, Sep. 2004.

[40] H. Yoo and J. Jeong, "Direction-oriented interpolation and its application to de-interlacing," IEEE Trans. Consum. Electron., vol. 48, no. 4, pp. $954-962$, Apr. 2002

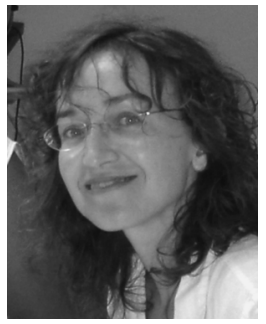

Coloma Ballester received the Licenciatura degree in mathematics from Barcelona University (UAB), Barcelona, Spain, and the Ph.D. degree in computer science from the University of Illes Balears, Spain, in 1995.

She is currently an Associate Professor at the Pompeu Fabra University, Barcelona. Her research interests include image processing and computer vision.

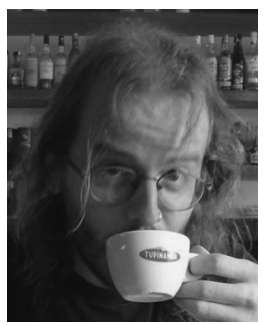

Marcelo Bertalmío received the B.Sc. and M.Sc. degrees from the Universidad de la Republica, Uruguay, in 1996 and 1998, respectively, and the Ph.D. degree from the University of Minnesota, Minneapolis, in 2001.

$\mathrm{He}$ is an Associate Professor at the Universitat Pompeu Fabra, Barcelona, Spain.

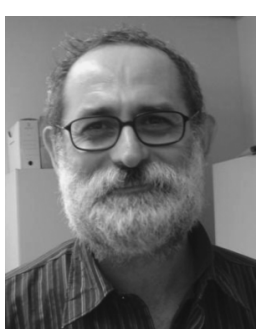

Vicent Caselles (A'94) received the Licenciatura and $\mathrm{Ph} . \mathrm{D}$. degrees in mathematics from Valencia University, Spain, in 1982 and 1985, respectively.

Currently, he is a Professor at the Universitat Pompeu Fabra, Barcelona, Spain. His research interests include image processing, computer vision, and the applications of geometry and partial differential equations to both fields.

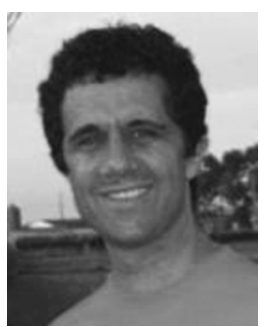

Luis Garrido received the Engineering Degree in telecommunications and the Ph.D. degree from the Universitat Politécnica de Catalunya (UPC), Spain, in 1996 and 2002, respectively.

In 2003, he joined the Image Processing Group in the Universitat Pompeu Fabra, Barcelona, Spain, where he currently holds a Ramon y Cajal position. His current interests are focused on contrast invariant motion estimation, multigrid techniques, and region-based analysis of images. 


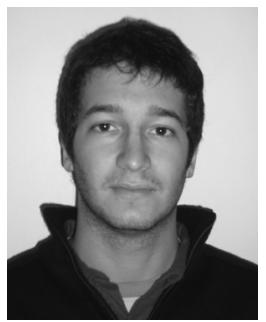

Adrián Marques received the degree in computer engineering from the Universidad de la Republica, Uruguay, in February 2006.

He worked in the Image Processing Group at Pompeu Fabra University, Barcelona, Spain, on video processing research. His other research interests include computer vision and artificial intelligence.

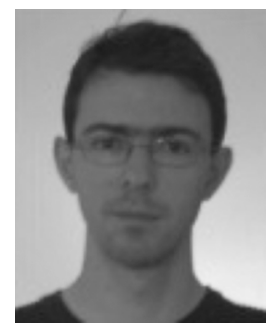

Florent Ranchin received the Engineer Diploma from the Ecole Centrale Marseille, France, in 2001. Under the supervision of Prof. F. Dibos, he received the $\mathrm{Ph} . \mathrm{D}$. degree in applied mathematics on the problems of segmentation and tracking in video sequences from Paris Dauphine University, France, in 2004

From September 2005 to August 2006, he occupied a postdoctorate position at the Department of Technology of Pompeu Fabra University, Barcelona, Spain. Currently, he occupies an engineering-research position at the LCEI, LIST of the CEA Saclay, France. His research interests include video segmentation, 2-D or 3-D tracking, variational methods, level sets, and particle filtering. 